\title{
Enhanced B-wavelets via mixed, composite packets
}

\author{
J. D. B. Nelson, Member, IEEE,
}

\begin{abstract}
A modified $B$-wavelet construction with enhanced filter characteristics is considered. The design comprises a superposition of tessellated, integer dilated, 'sister' wavelet functions. We here propose a cascaded filter-bank realisation of this wavelet family together with some notable extensions. We prove that modifications of low-order members exist in the multiresolution subspace spanned by the half-translates of the original wavelets and hence that the resulting modified wavelet coefficients can be computed as convolutions of the undecimated original wavelet coefficients. Finite impulse response filters are thus designed and incorporated into a $B$-wavelet packet architecture such that the mainlobe-to-sidelobe ratio of the resulting wavelet filter characteristic is improved. This is achieved by designing the filters so that zeros are introduced near to the maxima of the harmonics. It is shown that the numbers of zeros can be balanced with the length of the corresponding filters by controlling the 'modification order'. Several constructions are presented. We prove that two such constructions satisfy the perfect reconstruction property for all orders. The resulting modified wavelets preserve many of the properties of the original $B$-wavelets such as differentiability, number of vanishing moments, symmetry, anti-symmetry, finite support, and the existence of a closed form expression.
\end{abstract}

Index Terms $-B$-wavelets, wavelet packets, filter characteristic

\section{INTRODUCTION}

A DVANCES in modern linear and quadratic optimisation, and the subsequent popularity of the 'sparse way' [12] afforded by lasso, compressive sensing, and other such approaches, has generated continued interest in adaptive signal representations such as wavelet packets and other related non-standard wavelet transform architectures. A key, early approach to adapt a packet representation to signals was the best basis method of Coifman and Wickerhauser [8]. This employed an entropy-driven criterion to find the 'best basis' for a given signal. As such, the method explicitly incorporated a flexible, data-driven approach to signal processing into the multiresolution cascade structure of the wavelet packet transform itself. Various adaptive algorithms have since exploited the sparsifying properties of wavelet bases, frames, packets, and dictionaries and there now exist many extensions and generalisions of best basis principles such as the matching pursuit algorithm of Mallat and Zhang [13], the basis pursuit of Chen et al [5], the wavelet footprints of Dragotti and Vetterli [10], and more recently, the scattering transform of Andén and Mallat [1].

Indeed, basic developments in wavelet packet construction are still ongoing. The very interesting work of Weickert et al [22] incorporates Kingsbury's approximate shift invariant dualtree complex wavelets [11], [17] into a filter-swapping, packet

\footnotetext{
J. D. B. Nelson is with the Department of Statistical Science, University College London, UK e-mail: (see http://http://www.ucl.ac.uk/statistics/people/jamesnelson).
}

architecture. The result extends wavelet packets to analytic wavelet packets. In a more general setting, Chebira et al [4] recently constructed a fusion frame version of wavelet packets.

The wavelet packet transform effects a tiling of the frequency domain which is finer than the octave-based analysis offered by the usual fast wavelet transform. This is achieved by applying low- and high-pass filters to the detail coefficients. Instead of focussing explicitly on tilings and partitions of the time-frequency plane, we are here more interested in the filter characteristic shape of the resulting decomposition. It is well known that the decay of the filter characteristic determines the number of vanishing moments and therefore the ability of the wavelet to represent most signals in a sparse manner. Similarly, and irrespective of any notion of sparsity, in some applications such as digital communications [9] and antenna design [6], the mainlobe-to-sidelobe ratio of the filter characteristic is widely accepted as a key measure of filter efficacy.

Whilst Bayram and Selesnick [3] introduced wavelets that possess a higher, and constant, 'Q-factor' (ratio of centre frequency to bandwidth) little attention has been paid to exploiting extended constructions such as packets to explicitly improve the filter characteristic shape. We explore this idea with B-wavelets, first proposed by Chui [7] as the natural wavelet companions of the classical cardinal $B$-splines [16].

Wavelet construction from $B$-splines is attractive because the spline functions are very simple. Their impulse responses are merely autoconvolutions of the characteristic function on the unit interval. As such, they have finite support, a closed form expression in both time and frequency, and the number of autoconvolutions explicitly controls the number of vanishing moments. Indeed, as Unser et al [20] have so skillfully illustrated with their Riesz-Laplace construction, there is still much continued interest in wavelets constructed from $B$-splines.

The frequency decay of the $B$-wavelets is related to the order of smoothness which, of course, in turn dictates the number of vanishing moments [2]. However, Nelson [14] constructed a modified $B$-wavelet family and illustrated that, at least in some local frequency band, higher vanishing moments (i.e. higher spline order) are not necessarily required to obtain a more favourable frequency characteristic decay. This modified family amends the $B$-wavelets by forming a superposition of tessellated, integer dilated, 'sister' wavelet functions such that the filter characteristics are enhanced. However, it is not clear how such a family could be implemented via finite impulse response (FIR) filters in a multiresolution analysis, cascaded fliterbank framework. We revisit this construction and here show that a dilated subset of this family can be realised by applying additional finite impulse response filters to ordinary $B$-wavelet detail coefficients in a wavelet packet architecture. This results in a packet scheme which is 'mixed', in the sense that it contains more than one set of low- and high- 
pass filters, and which is 'composite' in the sense that the usual downsampling operation that immediately follows the high-pass filter is delayed until after the application of the additional filters.

In contrast to the $M$-band wavelets [15], which decomposes the signal into $M$-many different bands at each scale level, from Figure 3 it can be seen that the proposed construction is 'folded' into the usual, more common, 2-band framework.

Further modifications are introduced to our mixed, composite design in order to generalise the result to satisfy perfect reconstruction and all orders of smoothness. We thus arrive at a construction which accommodates the modified B-wavelets inside the usual 2-band fast wavelet architecture. The resulting wavelets share the same elegantly simple scaling functions, and preserve many of the properties, of the unmodified $B$ wavelets such as differentiability, smoothness, number of vanishing moments, symmetry, anti-symmetry, finite support, perfect reconstruction, and the existence of a closed form expression (for both the impulse response and filter characteristic). In addition, the wavelet characteristic function is enhanced without resorting to higher vanishing points.

In Section II], we offer a brief introduction to the modified $B$-wavelets proposed in [14]. Theoretical results are presented in Section III which motivate a wavelet packet architecture as a means of modifying the $B$-wavelet family such that the mainlobe-to-sidelobe ratios are improved. However, it is also shown that only the least smooth $B$-wavelets can be realised in such a packet scheme. In Section IV] extensions to smoother, higher-order wavelets and perfect reconstruction wavelets are presented.

\section{ENHANCED $B$-WAVELET FAMILIES}

The enhanced wavelet construction in [14] takes an existing wavelet $\psi$, supported on $T:=[0, t), t \in \mathbb{N}_{*}$, say, and adds weighted, dilated, and translated versions of itself, viz.

$$
\psi^{* N}:=\sum_{n=1}^{N} \gamma_{n} \mathcal{T}_{n} \psi,
$$

where we define the family of mappings $\mathcal{T}_{n}: T \mapsto T$ over the support of $\psi$ by

$$
\mathcal{T}_{n} \psi:=\sum_{k=0}^{\left(d_{n}-1\right) t} \psi\left(d_{n} \cdot-k\right),
$$

where $\left\{d_{n}\right\}=\{1,3,5,7,11,13,15, \ldots\}$ is the sequence of ascending, odd, square-free integers (i.e. with distinct divisors, where no prime factor is repeated). Throughout we define

$$
d:=\prod_{n=1}^{N} d_{n}
$$

For the $M$ th order $B$-wavelet, the weights $\left\{\gamma_{n}\right\}$ are

$$
\gamma_{n}=\frac{\mu(n) d_{n}^{1-M}}{(2 M-1)\left(d_{n}-1\right)+1},
$$

where $\mu$ is the Möbius arithmetic function, defined by

$$
\mu(n):= \begin{cases}1, & n=1 \\ (-1)^{k}, & n \text { is the product of } k \text { distinct primes } \\ 0, & \text { otherwise }\end{cases}
$$

Example 2.1: For $M=1, N=3$, we have $d_{1}=1, d_{2}=$ $3, d_{3}=5, d=15$ which gives an enhanced Haar wavelet defined by

$$
\begin{aligned}
\psi_{1}^{* 3}:= & \psi_{1}-\frac{1}{3}\left(\psi_{1}(3 \cdot)+\psi_{1}(3 \cdot-1)+\psi_{1}(3 \cdot-2)\right) \\
& -\frac{1}{5}\left(\psi_{1}(5 \cdot)+\ldots+\psi_{1}(5 \cdot-4)\right) .
\end{aligned}
$$

Unfortunately, although Nelson [14] showed that this 'family of family' of wavelets (over $M$ and $N$ ) has an associated fast integral wavelet transform, there is no obvious way to implement the discrete transform with a multiresolution analysis, critically sampled, filter-bank such as the fast wavelet, or packet, transforms. Instead, we consider a dilated version of this construction, namely

$$
\psi_{M}^{N}:=\psi_{M}^{* N}\left(\frac{1}{d} \cdot\right) .
$$

The impulse responses and filter characteristics of the first few members of this family are plotted in Figure 1. For illustrative purposes, the impulse responses have been dilated to give equal support to the modifications $(N=2,3)$. Likewise, the filter characteristics for $N=2,3$ are dilated to help illustrate that the modified wavelets do indeed mitigate sidelobe energy in a local frequency band about the fundamental frequency of the wavelet. Since the aim of these wavelets is to improve the mainlobe-to-sidelobe ratios, the precise amount of dilation is not of primary importance; however we note that for this family the dilation by $d^{-1}=\prod_{n=1}^{N} d_{n}^{-1}$ guarantees that the

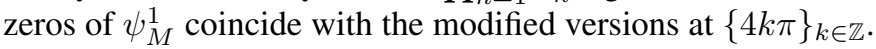

For the case $N=2$, the sidelobe energy at the first harmonic is attenuated. For $N=3$, the first and second harmonics are attenuated. From [14] (see Theorem 3.2 therein), this results from the fact that the filter characteristic energy is zeroed at $2 k \pi / d$ for all $k \in d_{n} \mathbb{N}, n=2, \ldots, N$. It is visibly evident that, as the order of modification $N$ is increased, the mainlobe-to-sidelobe ratios are improved. Hence, the modified wavelets constitute a doubly-indexed family of wavelets $\left\{\psi_{M}^{N}\right\}$ such that the mainlobe-to-sidelobe ratios are controlled by the modification order $N$ and the number of vanishing moments is controlled by smoothness order $M$.

\section{COnstruction}

A key relationship in the standard multiresolution analysis is the 'wavelet equation' which relates the wavelet function to the scaling function $\phi_{M}$ via $\psi_{M}=\sqrt{2} \sum_{k} h_{1}[k] \phi_{M}(2 \cdot-k)$ and where the scaling function satisfies the refinement equation $\phi_{M}=\sqrt{2} \sum_{k} h_{0}[k] \phi_{M}(2 \cdot-k)$. If two such finite sequences exist then a fast wavelet transform architecture can be realised.

In this section Theorem 3.3, below, establishes the result that the modified wavelets lie in the subspace spanned by the $B$-wavelet scaling functions. The result, which applies to all smoothness orders, affords an FIR implementation of the modified wavelet transform whereby an extra filter-bank is applied to the (undecimated) scaling coefficients. However, it is later shown that this extra processing sub-block cannot satisfy FIR perfect reconstruction. This motives an 


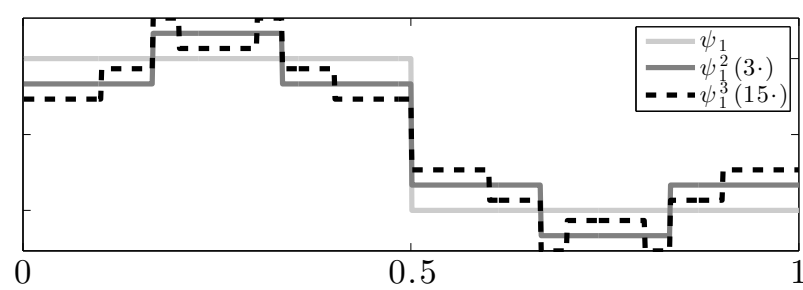

(a) $M=1$

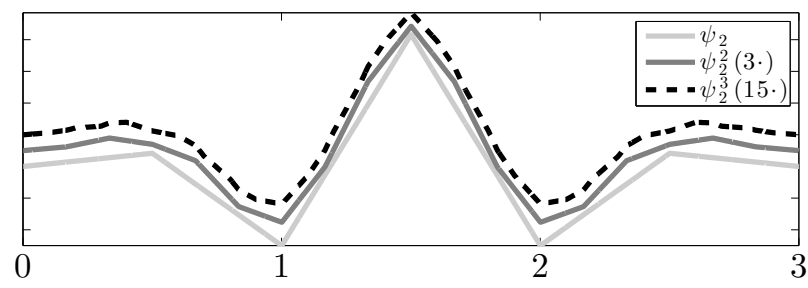

(c) $M=2$

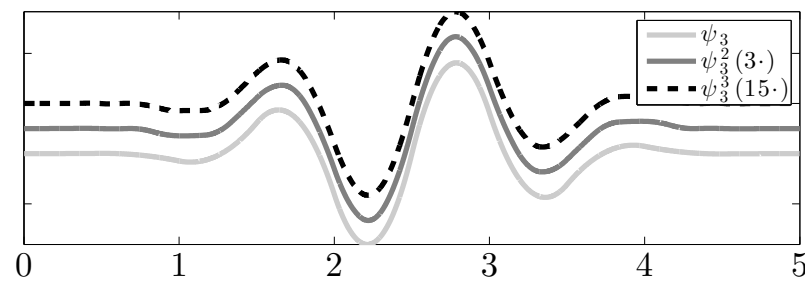

(e) $M=3$

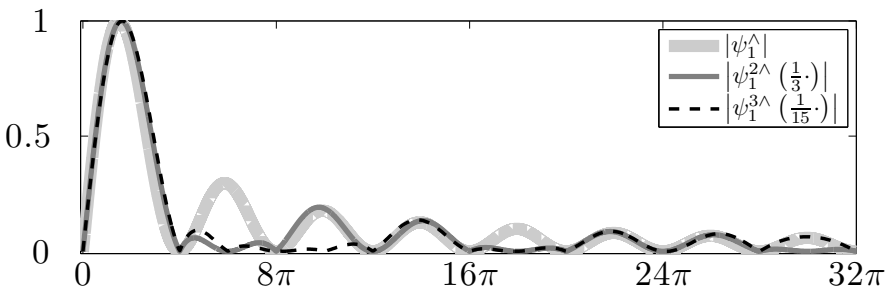

(b) $M=1$

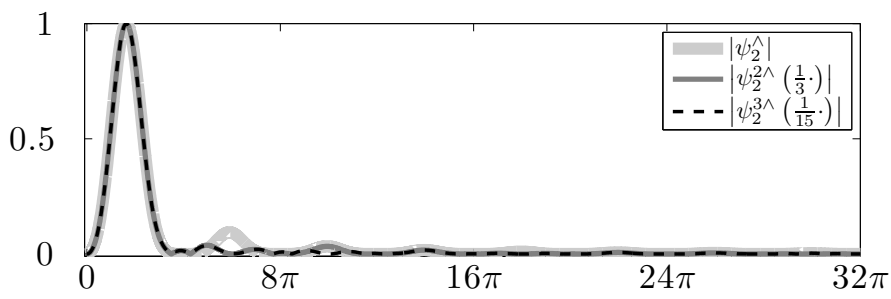

(d) $M=2$

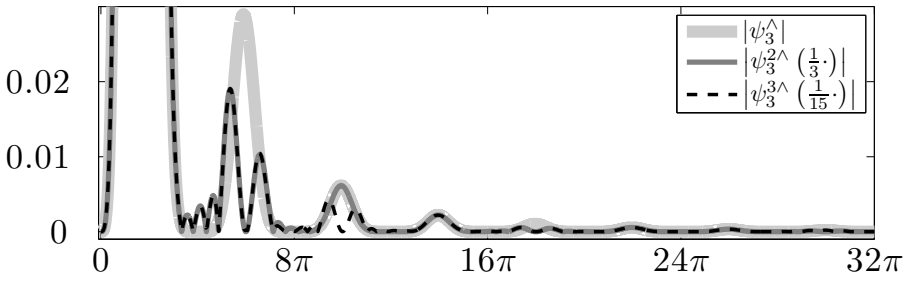

(f) $M=3$

Fig. 1. The impulse responses (a,c,e) and filter characteristics (b,d,f) of the $B$-wavelet $\psi_{M}$ and modified $B$-wavelet $\psi_{M}^{N}$ for 'smoothness' orders $M=1,2,3$ and modification orders $N=1,2,3$. The modified wavelets with $N=2$ attenuate the the first harmonic by introducing zeros at $6 k \pi$; likewise the wavelets with $N=3$ attenuate the the first and second harmonics by introducing zeros at $6 k \pi$ and $10 k \pi$. Note that the impulse response functions for $M=2,3$ have been shifted vertically and the vertical axis of sub-figure (f) has been chosen for illustrate purposes (the main lobe magnitude is 1 ).

alternative strategy. To this end, Theorem 3.4 below, shows that low-order members of the modified family exist in the multiresolution subspace spanned by the half-translates of the original wavelets. Together with Proposition 3.5 provides a means by which the modified wavelet coefficients can be computed as convolutions of the undecimated original wavelet coefficients. In other words, we show that the family $\left\{\psi_{1}^{N}\right\}$ of wavelets with smoothness order $M=1$ can be realised with FIR filters, applied in a wavelet packet-like architecture where an additional analysis/synthesis processing sub-block is added to the detailed coefficient branch of the usual filterbank architecture. Although notation is defined in context throughout, some of the key terms are collected together in Table \ for convenience.

We will first need to establish the fact that a dilated $B$-spline can be written as a superposition of non-dilated $B$-splines.

Lemma 3.1: Let $\phi_{M}:=\phi_{1} * \phi_{M-1}$ denote the $M$ th order $B$-spline with $\phi_{1}(x):=1$ if $x \in[0,1)$ and zero otherwise. Then, for $n \in \mathbb{N}_{*}$

$$
\phi_{M}\left(\frac{1}{n} \cdot\right)=\frac{1}{n^{M-1}} \sum_{k} \mathbb{1}_{n}^{M}[k] \phi_{M}(\cdot-k),
$$

where $\mathbb{1}_{n}^{M}$ is the $M$-fold convolution of the sequence of $n$ many ones, $\mathbb{1}_{n}$.
TABLE I

NOTATION

\begin{tabular}{l|l} 
Term & Definition \\
\hline$\phi_{M ; j, k}$ & $\begin{array}{l}B \text {-spline scaling function with smoothness order } M \text { at the } \\
j \text { th finest scale level and } k \text { th integer translate, cf. Eqn. 57 }\end{array}$ \\
$\psi_{M ; j, k}$ & $\begin{array}{l}B \text {-wavelets with smoothness order } M, \text { cf. Eqn. 6 } \\
\text { modified } B \text {-wavelets (modification order } N \text {, smoothness }\end{array}$ \\
$\psi_{M ; j, k}^{N}$ & $\begin{array}{l}\text { order } M \text { ) } \\
M \text {-fold convolution of } n \text {-many ones }\end{array}$ \\
$\mathbb{1}_{n}^{M}$ & $\begin{array}{l}\text { upsampling operator (Definition 3.2 } \\
\uparrow \cdot \\
i \text { th square-free odd integer }\end{array}$ \\
$d_{i}$ & $B$-wavelet scaling and wavelet FIR filters \\
$h_{0}, h_{1}$ & $\begin{array}{l}\text { proposed mixing filters which act upon the undecimated } \\
\text { detail }\end{array}$ \\
$h_{2}, h_{3}$ & wavelet coefficients, from $j$ th finest scale level, of the \\
$d_{j}^{(i)}$ & $\begin{array}{l}\text { schemes presented in Fig } 2 \text { (for } i \in\{0,1\} \text { ) and Fig. } 3 \text { (for } \\
i \in\{2,3\} \text { ). }\end{array}$
\end{tabular}

Proof We have

$$
\phi_{2}\left(\frac{1}{n} \cdot\right)=(\phi * \phi)\left(\frac{1}{n} \cdot\right)=\int \phi(x) \phi\left(\frac{1}{n} \cdot-x\right) \mathrm{d} x .
$$

Now, using the identity $\phi\left(n^{-1} \cdot\right)=\sum_{k} \mathbb{1}_{n}[k] \phi(\cdot-k)$ twice and making substitutions (see Appendix) we have that

$$
\phi_{2}\left(\frac{1}{n} \cdot\right)=\frac{1}{n} \sum_{k, \ell} \mathbb{1}_{n}[k] \mathbb{1}_{n}[\ell-k] \int \phi(x) \phi(\cdot-\ell-x) \mathrm{d} x
$$




$$
=\frac{1}{n} \sum_{\ell}\left(\mathbb{1}_{n} * \mathbb{1}_{n}\right)[\ell](\phi * \phi)(\cdot-\ell),
$$

which proves the result for $M=2$. A simple inductive argument is used (see Appendix) to complete the proof.

Definition 3.2: Define the upsampling operator $\uparrow$ by

$$
((\uparrow n) h)[k]:= \begin{cases}h[k / n], & \text { if } k \in n \mathbb{Z} \\ 0, & \text { otherwise }\end{cases}
$$

Theorem 3.3: As above, let $\psi_{M}^{N}$ denote the $(M, N)$-order modified wavelet and let $\phi_{M}$ denote $M$ th order $B$-spline. Then,

$$
\psi_{M}^{N}=\sum_{k=0}^{K} g[k] \phi_{M}(2 \cdot-k),
$$

with the finitely supported, square summable sequence

$$
g=\sum_{n=1}^{N} \gamma_{n}\left(\mathbb{1}_{d_{n}^{\sim}}^{M} *\left(\uparrow d_{n}^{\sim}\right) h_{1} *\left(\uparrow 2 d_{n}^{\sim}\right) \mathbb{1}_{d_{n}^{(M)}}\right),
$$

where $d_{n}^{\sim}:=d / d_{n}$.

Proof The result follows by noting that the definition of the modified wavelet is equivalent to a filtering on a dilated version of $\psi_{M}$ which, itself, can be written in terms of a filtering of a dilated version of $\phi_{M}(2 \cdot)$ which, courtesy of Lemma 3.1 can be written as a filtering of $\phi_{M}(2 \cdot)$. To start with we have, by definition, that

$$
\psi_{M}^{N}=\sum_{n} \gamma_{n}\left(\mathcal{T}_{n} \psi\right)\left(\frac{1}{d} \cdot\right)
$$

But, using $d_{n}^{\sim}:=d / d_{n}$, we note that

$$
\begin{aligned}
\left(\mathcal{T}_{n} \psi\right)\left(\frac{1}{d} \cdot\right) & =\sum_{k=0}^{\left(d_{n}-1\right) t} \psi_{M}\left(\frac{1}{d_{n}^{\sim}} \cdot-k\right) \\
& =\sum_{k} \mathbb{1}_{d_{n}^{(M)}}[k] \psi_{M}\left(\frac{1}{d_{n}^{\sim}} \cdot-k\right)
\end{aligned}
$$

where we have set $d_{n}^{(M)}:=\left(d_{n}-1\right) t+1$ and where we recall that $t=\left|\operatorname{supp} \psi_{M}\right|=2 M-1$ for $B$-wavelets. From Lemma 3.1, we use the result

$$
\phi_{M}\left(\frac{2}{d_{n}^{\sim}} \cdot\right)=\sum_{\ell} \mathbb{1}_{d_{n}^{\sim}}^{M}[\ell] \phi_{M}(2 \cdot-\ell)
$$

and the wavelet equation to find that

$$
\begin{aligned}
\psi_{M}\left(\frac{1}{d_{n}^{\sim}} \cdot\right) & =\sum_{k} h_{1}[k] \phi_{M}\left(\frac{2}{d_{n}^{\sim}} \cdot-k\right) \\
& =\sum_{k, \ell} h_{1}[k] \mathbb{1}_{d_{n}^{\sim}}^{M}[\ell] \phi_{M}\left(2 \cdot-\ell-d_{n}^{\sim} k\right) \\
& =\sum_{\ell}\left(\mathbb{1}_{d_{\tilde{n}}^{\sim}}^{M} *\left(\uparrow d_{n}^{\sim}\right) h_{1}\right)[\ell] \phi_{M}(2 \cdot-\ell)
\end{aligned}
$$

Gathering equations (2), (3), and (4), we have

$\psi_{M}^{N}=\sum_{n=1}^{N} \sum_{k, \ell} \gamma_{n} \mathbb{1}_{d_{n}^{(M)}}[k]\left(\mathbb{1}_{d_{n}^{\sim}}^{M} *\left(\uparrow d_{n}^{\sim}\right) h_{1}\right)[\ell] \phi_{M}\left(2 \cdot-\ell-2 d_{n}^{\sim} k\right)$,
Substituting $\ell^{\prime}=\ell+2 d_{n}^{\sim} k$ gives

$$
\psi_{M}^{N}=\sum_{\ell} \sum_{n=1}^{N} \gamma_{n}\left(\mathbb{1}_{d_{n}^{\sim}}^{M} *\left(\uparrow d_{n}^{\sim}\right) h_{1} *\left(\uparrow 2 d_{n}^{\sim}\right) \mathbb{1}_{d_{n}^{(M)}}\right)[\ell] \phi_{M}(2 \cdot-\ell)
$$

which is of the desired form.

Theorem 3.3 implies that the modified wavelets $\psi_{M}^{N}$ lie in the span of $\phi_{M}(2 \cdot)$. As such, it may seem natural to simply obtain the modified wavelet coefficients $\left\langle f, \psi_{M}^{N}\right\rangle$ by employing $h_{0}$ and $g_{0}=2^{-1 / 2} g$ in a standard two-channel filter bank. However, in order for FIR perfect reconstruction to be satisfied, and hence the transform to be invertible, the analysis filters $h_{0}$ and $g_{0}$ must be complementary; equivalently, a necessary and sufficient condition for perfect reconstruction to hold is that $P(z)+P(-z) \propto z^{-2 \ell-1}$, for some $\ell \in \mathbb{Z}$ where the polynomial $P$ is defined as $P(z):=H_{0}(z) G_{0}(-z)$ and where $H_{0}$ and $G_{0}$ are the z-transforms of the filter sequences $h_{0}$ and $g_{0}$ (cf. Fact 3.1 and Equation (41) on page 2218 of Vetterli [21]). However, clearly, this beaks down in even the most simple non-trivial case $(M=1, N=2)$ where we find that $P(z)-P(-z)=2 z^{-6}-2 z^{-4}-2 z^{-2}+2$.

Alternative architectures are thus motivated. One could, for example, consider the architecture depicted in Figure 2 which resembles a packet-like scheme. First, the usual low-pass filter $h_{0}$ is applied to the scaling wavelet coefficient $c_{j-2}$ at the $(j-2)$ th finest scale level, with downampling, to obtain $c_{j-1}$, the scaling wavelet coefficient at the $(j-1)$ th level. Then the filter $g_{0}$, say, is applied before downsampling. The result, $d_{j}^{(0)}$, is the modified wavelet coefficient. In order to realise a filterbank implementation with perfect reconstruction we are then free to construct a complementary filter $g_{1}$ which must necessarily exist along with the duals $g_{0}^{\sim}, g_{1}^{\sim}$ [18]. Note also that this result holds for all $M$ and $N$ because Theorem 3.3 states that there exists finite length $g_{0}=2^{-1 / 2} g$ such that

$$
\psi_{M ; j, k}^{N}=\sum_{n} g_{0}[n] \phi_{M ; j-1,2 k+n},
$$

where here, and throughout, we define

$$
\begin{aligned}
& \phi_{M ; j, k}:=2^{-j / 2} \phi_{M}\left(2^{-j} \cdot-k\right), \\
& \psi_{M ; j, k}^{N}:=2^{-j / 2} \psi_{M}^{N}\left(2^{-j} \cdot-k\right) .
\end{aligned}
$$

It follows immediately that

$$
\begin{aligned}
d_{j}^{(0)}[k]:=\left\langle f, \psi_{M ; j, k}^{N}\right\rangle= & \left(g_{0}^{T} * c_{j-1}\right)[2 k] \\
=: & (\downarrow 2)\left(g_{0}^{T} * c_{j-1}\right)[k],
\end{aligned}
$$

where superscript $T$ denotes a (temporal) flip, $g_{0}^{T}:=g_{0}[-\cdot]$, and where $c_{j}:=\left\langle f, \phi_{M ; j, k}\right\rangle$. The superscript 0 on the right hand side refers to the result of filtering $c_{j-1}$ by the sequence $g_{0}$. Likewise, the coefficients $d_{j}^{(1)}=(\downarrow 2)\left(g_{1}^{T} * c_{j-1}\right)[k]$ are the result of filtering $c_{j-1}$ by the complementary filter $g_{1}$.

Of course, Figure 2 is markedly different from the conventional packet architecture which simply applies a further low- and high-pass filter bank after the usual high-pass and downsampling operations. Instead, Figure 2 applies two filters $g_{0}$ and $g_{1}$ immediately after the low-pass filter $h_{0}$ (without downsampling first). However as discussed later in Section IV-B it turns out that, as defined, there do not exist any dual 


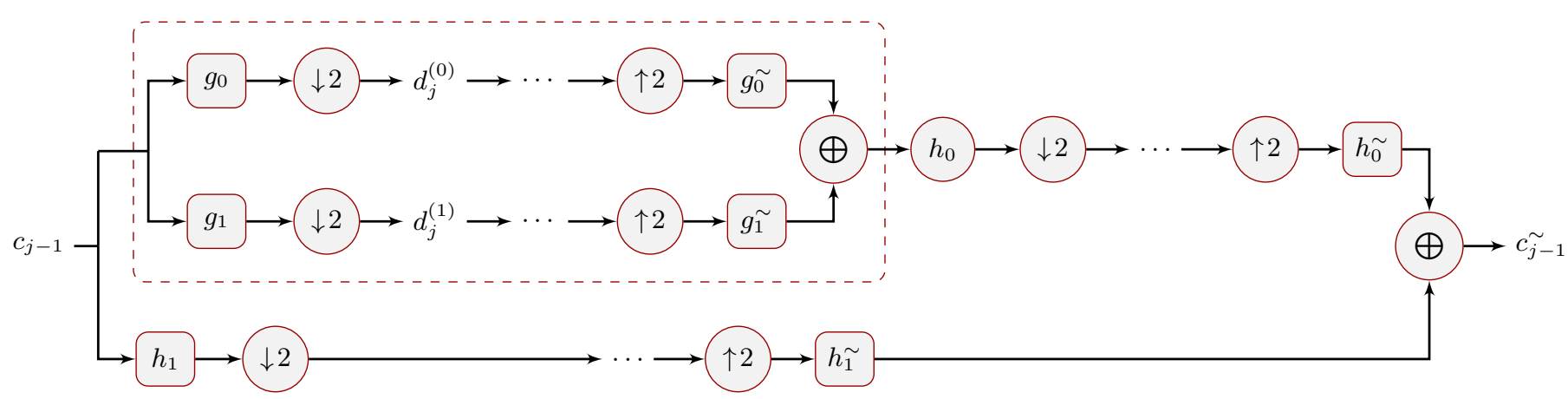

Fig. 2. A mixed, composite wavelet packet architecture. In the analysis phase, the undecimated original scaling (a.k.a approximation) coefficients are filtered by $g_{0}$ and (if it exists) its complementary filter $g_{1}$ and then downsampled by two to get the modified wavelet coefficients $d_{j}^{(0)}$ (together with $d_{j}^{(1)}$ ), say. For synthesis/reconstruction, these coefficients are upsampled before the dual filters (if they exists) $g_{0}^{\sim}$ and $g_{1}^{\sim}$ are applied. This processing sub-block (delimited by the dashed box) can be applied to any subset of nodes in the usual fast wavelet transform tree. Note that the removal of the sub-block simply returns the usual fast (decimated) wavelet transform analysis/synthesis diagram and that the scaling coefficient at $c_{j}$ can be obtained, in the usual manner, by applying the scaling filters $h_{0}$ to $c_{j-1}$ and downsampling.

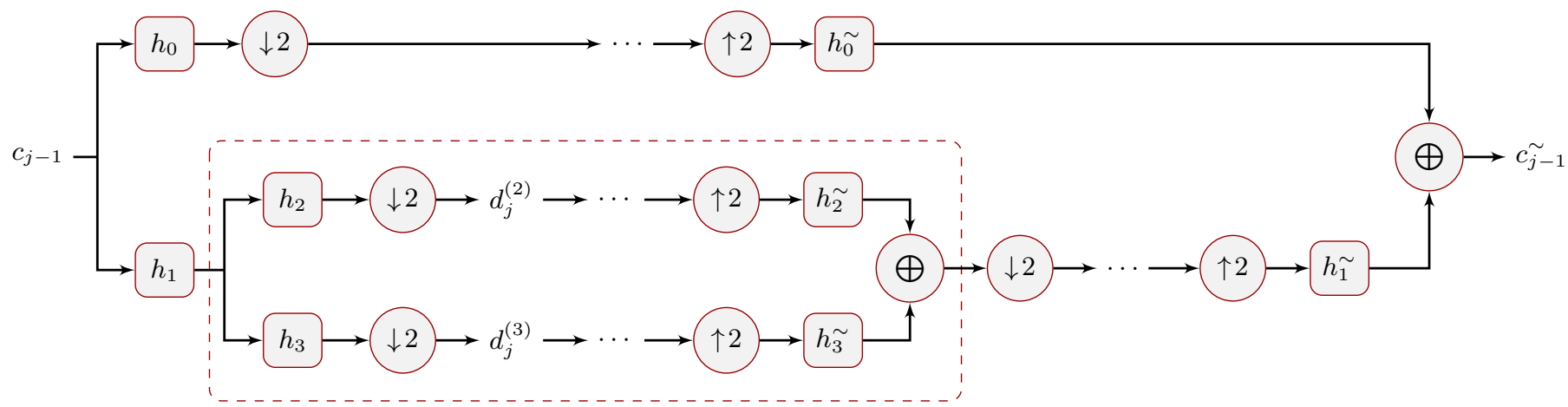

Fig. 3. A mixed, composite wavelet packet architecture. In the analysis phase, the undecimated original wavelet detail coefficients (as opposed to the scaling coefficients in Figure 2) are filtered by $h_{2}$ and (if it exists) its complementary mirrored filter $h_{3}$ and then downsampled by two to get the modified wavelet coefficients $d_{j}^{(2)}$ (together with $d_{j}^{(3)}$ ), say. For synthesis/reconstruction, these coefficients are upsampled before the dual filters (if they exist) $h_{2}^{\sim}$ and $h_{3}^{\sim}$ are applied. This processing sub-block (delimited by the dashed box) can be applied to any subset of nodes in the usual fast wavelet transform tree. Note that the removal of the sub-block simply returns the usual fast (decimated) wavelet transform analysis/synthesis diagram and that the scaling coefficient at $c_{j}$ can be obtained, in the usual manner, by applying the scaling filters $h_{0}$ to $c_{j-1}$ and downsampling.

filters $g_{0}^{\sim}, g_{1}^{\sim}$ (for the wavelet $\psi_{M}^{N}$ ) such that the synthesis part of Figure 2 holds. (For example, for $M=1, N=2$, the ztransform of the filter $g_{0}=[1,2,1,-1,-2,-1]$ factorises as $G_{0}(z)=(z+1)^{2}(1-z)\left(1+z+z^{2}\right)$ which clearly has zeros in pairs at \pm 1 and therefore breaks the conditions in Proposition 4.6 in Section 4 below- cf. Proposition 4.2, page 2222 of Vetterli [21].)

Instead, the following theorem provides some way forward, albeit for the single case $M=1$. It suggests that the modified wavelet coefficients can be realised by working with the high-pass, rather than low-pass coefficients. This result is consolidated by Proposition 3.5 and Corollary 3.6 below which explicitly find the relevant filter coefficients that make this possible.

Theorem 3.4: Let $\psi_{M}^{N}$ denote the $(M, N)$-order modified wavelet and let $K \in \mathbb{N}$. Then, the condition

$$
\psi_{M}^{N} \in \operatorname{span}\left\{\psi_{M}\left(\cdot-\frac{1}{2} k\right), 0 \leq k \leq K\right\}
$$

only holds for $M=1$.
Proof From Theorem 3.3 we have that

$$
\psi_{M}^{N}=\sum_{\ell} \sum_{n}\left(\alpha_{n} *\left(\uparrow d_{n}^{\sim}\right) h_{1}\right)[\ell] \phi_{M}(2 \cdot-\ell) .
$$

In order for $\psi_{M}^{N}$ to satisfy condition 77 , there must exist a square summable, FIR $a$ such that

$$
\sum_{n}\left(\alpha_{n} *\left(\uparrow d_{n}^{\sim}\right) h_{1}\right)=a * h_{1},
$$

i.e. there must exist a collection of FIRs $a_{n} \in \ell_{2}(\mathbb{Z}), 1 \leq n \leq$ $N$ such that

$$
\left(\uparrow d_{n}^{\sim}\right) h_{1}=a_{n} * h_{1} .
$$

Taking Z-transforms of both sides $\left(a_{n} \mapsto A_{n}, h_{1} \mapsto H_{1}\right)$, we see that this is equivalent to the existence of Laurent polynomials $A_{n}(\cdot)$ such that

$$
A_{n}(z)=\frac{H_{1}\left(z^{d_{n}^{\tilde{n}}}\right)}{H_{1}(z)}, \quad z \in \mathbb{C} .
$$

However, this is only possible if all the roots of $H_{1}(z)$ have modulus one; otherwise, $H_{1}(z)$ does not divide into $H_{1}\left(z^{d_{n}^{\sim}}\right)$. 
The only $B$-wavelet with filter coefficients with such roots is the Haar wavelet $(M=1)$ with coefficients $H_{1}=[1,-1] / \sqrt{2}$.

Proposition 3.5: Let $\psi$ be a wavelet associated with a multiresolution analysis generated by the scaling function $\phi$. Suppose there exists $h_{2} \in \ell_{2}(\mathbb{Z})$ such that a modified version $\psi^{N}$ of the wavelet $\psi$ satisfies

$$
\psi_{j k}^{N}=\sum_{\ell} h_{2}[\ell] \psi_{j, k+\frac{1}{2} \ell} .
$$

Then

$$
\left\langle f, \psi_{j k}^{N}\right\rangle=(\downarrow 2)\left(h_{2}^{T} * h_{1}^{T} *\left\langle f, \phi_{j k}\right\rangle\right)[k] .
$$

$\underline{\text { Proof }}$

$$
\begin{aligned}
\psi_{j k}^{N} & =\sum_{\ell} h_{2}[\ell] \psi_{j, k+\frac{1}{2} \ell} \\
& =\sum_{\ell, n} h_{2}[\ell] h_{1}[n] \phi_{j-1, n+2 k+\ell} \\
& =\sum_{\ell}\left(h_{2} * h_{1}\right)[\ell] \phi_{j-1,2 k+\ell} \\
& =\sum_{\ell}\left(h_{2} * h_{1}\right)^{T}[\ell] \phi_{j-1,2 k-\ell} .
\end{aligned}
$$

Hence, for $f \in L_{2}(\mathbb{R})$, define $c_{j}[n]:=\left\langle f, \phi_{j n}\right\rangle$. Then,

$$
\begin{aligned}
\left\langle f, \psi_{j k}^{N}\right\rangle & =\sum_{\ell}\left(h_{2} * h_{1}\right)^{T}[\ell]\left\langle f, \phi_{j-1,2 k-\ell}\right\rangle \\
& =\left(h_{2}^{T} * h_{1}^{T} * c_{j-1}\right)[2 k] \\
& =(\downarrow 2)\left(h_{2}^{T} * h_{1}^{T} * c_{j-1}\right)[k] .
\end{aligned}
$$

Corollary 3.6: Let $\psi_{M ; j k}^{N}$ denote the $(M, N)$-order modified wavelet. Then

$$
\left\langle f, \psi_{1 ; j k}^{N}\right\rangle=(\downarrow 2)\left(h_{2}^{T} * h_{1}^{T} * c_{j-1}\right)
$$

with

$$
h_{2}[k]=\sum_{n} \gamma_{n} \mathbb{1}_{d_{\tilde{n}}^{\sim}}^{2} *\left(\uparrow 2 d_{n}^{\sim}\right) \mathbb{1}_{d_{n}}
$$

Proof From Theorem 3.3 we have that

$$
\psi_{1}^{N}=\sum_{\ell} \sum_{n}\left(\alpha_{n} *\left(\uparrow d_{n}^{\sim}\right) h_{1}\right)[\ell] \phi_{1}(2 \cdot-\ell),
$$

where $\alpha_{n}:=\gamma_{n} \mathbb{1}_{d_{n}} *\left(\uparrow 2 d_{n}^{\sim}\right) \mathbb{1}_{d_{n}}$. We make notationally explicit here the fact the filter sequence $h_{1}$ depends on $M$ by writing $h_{1}=h_{M, 1}$ and note that $\left(\uparrow d_{n}^{\sim}\right) h_{1,1}=\mathbb{1}_{d_{n}} * h_{1,1}$. Hence

$$
\begin{aligned}
\psi_{1}^{N} & =\sum_{\ell} \sum_{n}\left(\alpha_{n} * \mathbb{1}_{d_{\tilde{n}}} * h_{1,1}\right)[\ell] \phi_{1}(2 \cdot-\ell) \\
& =\sum_{k} \sum_{n}\left(\alpha_{n} * \mathbb{1}_{d_{\tilde{n}}}\right)[k] \psi_{1}\left(\cdot-\frac{1}{2} k\right) \\
& =: \sum_{k} h_{2}[k] \psi_{1}\left(\cdot-\frac{1}{2} k\right)
\end{aligned}
$$

Theorem 3.4 and Proposition 3.5 complete the proof.

Proposition 3.5 is important because, together with Theorem 3.4 it states that the modified discrete $B$-wavelet transform coefficients can be computed from the (undecimated) original
$B$-wavelet coefficients $h_{1}^{T} * c_{j-1}$ by first convolving with a flipped version of the FIR filter $h_{2}$ given by Corollary 3.6 and then downsampling by two. This architecture, shown in Figure 3, resembles the usual wavelet packet scheme more closely than that of Figure 2

\section{EXTENSIONS}

There now remain two immediate outstanding challenges. The first, addressed in Section IV-A is that, as defined above, the modified wavelets do not satisfy the architecture, described by Figure 3 , for $M>1$. The second challenge, addressed in Section IV-B is to satisfy this architecture for all $M$ such that perfect reconstruction is maintained-i.e. to ensure the existence of the dual filters $h_{2}^{\sim}$ and $h_{3}^{\sim}$.

\section{A. Extension to higher order B-wavelets}

A consequence of Theorem 3.4 and Proposition 3.5 is that, as defined, the modified wavelets only satisfy the architecture in Figure 3 for $M=1$. In other words, the identity

$$
\psi_{M}^{N}=\sum_{k} h_{M, 2}^{N}[k] \psi\left(\cdot-\frac{1}{2} k\right)
$$

only holds for $M=1$ and there does not exist any filters $h_{M, 2}^{N}$ such that 9 holds for $M>1$. This motivates us to extend the modified wavelets such that a result akin to Theorem 3.4 is satisfied for all smoothness orders $M$. Since the filter characteristics of all members of the $B$-wavelets (over all $M$ ) share the same zeros it is natural to consider using the same filter $h_{1,2}^{N}$ (for all $M$ ) to describe the modified wavelets in terms of the half-sample translates of the original B-wavelets; this alternative family takes the form

$$
\psi_{M}^{N, 1}:=\sum_{k} h_{M, 2}^{N, 1}[k] \psi_{M}\left(\cdot-\frac{1}{2} k\right),
$$

with, cf. Equation 8

$$
\begin{aligned}
h_{M, 2}^{N, 1} & :=h_{1,2}^{N} \\
& :=\sum_{n=1}^{N} \gamma_{n} \mathbb{1}_{d_{\tilde{n}}}^{2} *\left(\uparrow 2 d_{n}^{\sim}\right) \mathbb{1}_{d_{n}} .
\end{aligned}
$$

for all $M \in \mathbb{N}_{*}$. By Corollary 3.6 this modification can now be realised by FIR filter banks. Note that, for $M=1$, this alternative family is equivalent to the original modified family, namely $\psi_{1}^{N, 1} \equiv \psi_{1}^{N}$.

In Figure 4, the filter characteristics of $\psi_{M}^{N, 1}$ are plotted for the first few orders. Unfortunately, although the filter $h_{M, 2}^{N}$ does indeed introduce zeros near to the maxima of some of the sidelobes, the decay of the characteristic is insufficient to offer any benefit. Indeed, the sidelobes are even larger than the mainlobe. A straightforward way to counteract this effect is to use an autoconvolved version of $h_{M, 2}^{N}$ - i.e. to define

$$
\psi_{M}^{N, 2}:=\sum_{k} h_{M, 2}^{N, 2}[k] \psi_{M}\left(\cdot-\frac{1}{2} k\right),
$$

with $h_{M, 2}^{N, 2}:=*_{m=1}^{M} h_{1,2}^{N}$. The filter characteristics of an example of this family are also plotted in Figure 4. As an 
aside, it is also worth noting here that we we do not necessarily have to convolve $h_{1,2}^{N}$ with itself to guarantee a suitable decay. We could, instead, convolve it with some of its constituent convolutive factors as required. Since the filters $h_{1,2}^{N}$ form the

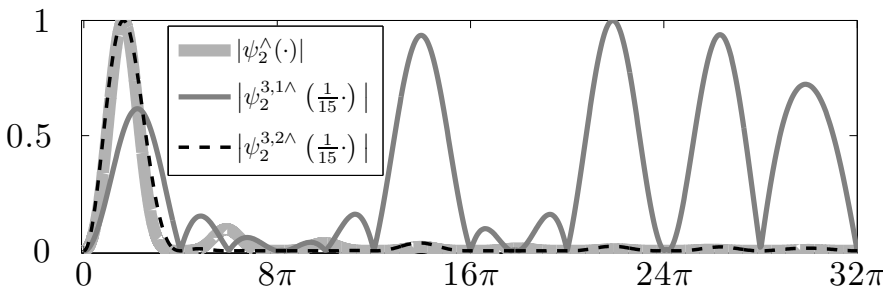

(a) $M=2, N=3$

Fig. 4. Filter characteristic comparison between $\psi_{2}^{3,1}$ and $\psi_{2}^{3,2}$. Although the dilated version of the wavelet $\psi_{2}^{3,1}$ possesses zeros in the 1st and 2nd harmonics of the B-wavelet, it has undesirable decay. On the other hand, the wavelet $\psi_{2}^{3,2}$ shares the zeros of $\psi_{2}^{3,1}$ and otherwise has a filter characteristic decay close to the original wavelet.

building blocks of the two families of wavelets $\left\{\psi_{M}^{N, 1}\right\}$ and $\left\{\psi_{M}^{N, 2}\right\}$ introduced thus far, we now present a simple example as follows.

Example 4.1: For $N=2$, we have $d_{1}=1, d_{2}=3, d_{1}^{\sim}=$ $3, d_{2}^{\sim}=1$ and, cf. Equation 8

$$
\begin{aligned}
h_{1,2}^{2} & =\sum_{n=1}^{2} \gamma_{n} \mathbb{1}_{d_{n}^{\sim}}^{2} *\left(\uparrow 2 d_{n}^{\sim}\right) \mathbb{1}_{d_{n}} \\
& ==\mathbb{1}_{3}^{2} *(\uparrow 6) \mathbb{1}_{1}-\frac{1}{3} \mathbb{1}_{1}^{2} *(\uparrow 2) \mathbb{1}_{3} \\
& =[1,2,3,2,1]-\frac{1}{3}[1,0,1,0,1]=\frac{2}{3}[1,3,4,3,1] .
\end{aligned}
$$

An alternative family of constructions, worthy of note, is obtained by eliminating all but $d_{1}=1$ and $d_{N}$ from the set $\left\{d_{1}, \ldots, d_{N}\right\}$ in the original definition given by Equation (1). This yields the wavelet $\left(\gamma_{1} \mathcal{T}_{1}+\gamma_{N} \mathcal{T}_{N}\right) \psi$ and $d=d_{N}$ which will attenuate the $(N-1)$ harmonic only (rather than all harmonics up to the $(N-1)$ th one). In a similar way to $\left\{\psi_{M}^{N}\right\}$, when dilated by $1 / d$, this alternative construction can be realised by FIR filters, namely

$$
\psi_{M}^{N, 3}:=\sum_{k} h_{M, 2}^{N, 3}[k] \psi_{M}\left(\cdot-\frac{1}{2} k\right)
$$

with

$$
\begin{aligned}
h_{1,2}^{N, 3} & :=\mathbb{1}_{d_{N}} *\left(\uparrow 2 d_{N}\right) \mathbb{1}_{1}+\gamma_{N} \mathbb{1}_{1}^{2} *(\uparrow 2) \mathbb{1}_{d_{N}} \\
& =\mathbb{1}_{d_{N}}^{2}+\gamma_{N}(\uparrow 2) \mathbb{1}_{d_{N}} .
\end{aligned}
$$

The filter characteristics of this family for $N=2,3,4$ and $M=1$ are plotted in Figure 5. We can see that the filter characteristic $\left|\psi_{1}^{N, 3 \wedge}\right|$ does indeed contain zeros such that the energy at the $(N-1)$ th harmonic (sidelobe) is attenuated. However, and similar to the case of the family $\psi_{M}^{N, 1}$, the decay of the filter characteristic is insufficient for $M>1$. Again, defining the higher order filters $h_{M, 2}^{N, 3}$ as autoconvolutions of the filter $h_{1,2}^{N, 3}$ can deal with this problem. However, removing the third harmonic (say) whilst leaving the first harmonic in tact does not appear to be particularly well-aligned with the main aim of designing wavelet filter realisations with improved mainlobe-to-sidelobe ratios. Happily, it turns out that combining $h_{M, 2}^{N, 3}$ with $h_{M, 2}^{N-1,3}$ for $N>2$ leads to a filter characteristic which suits our purpose quite well. We define:

$$
\psi_{M}^{N, 4}:=\sum_{k} h_{M, 2}^{N, 4}[k] \psi_{M}\left(\cdot-\frac{1}{2} k\right),
$$

with

$$
h_{M, 2}^{N, 4}:=h_{1,2}^{N-1,3} \underset{m=1}{\stackrel{M}{*}} h_{1,2}^{N, 3} .
$$

The (dilated) impulse responses and filter characteristics are plotted in figures 6 and 7. We can see that for $N=3$ the first 3 harmonics of the filter characteristic are attenuated and for $N=4$ the first five are attenuated. As the figure also illustrates, this feature also holds for higher order $(M>1)$ wavelets. The building blocks of this family are the filters $h_{1,2}^{N, 3}$. A couple of examples are given as follows

Example 4.2: For $N=2$, we have:

$$
h_{1,2}^{2,3}=\mathbb{1}_{3}^{2}-\frac{1}{3}(\uparrow 2) \mathbb{1}_{3}=h_{1,2}^{2}=\frac{2}{3}[1,3,4,3,1] .
$$

For $N=3$ :

$$
h_{1,2}^{3,3}=\mathbb{1}_{5}^{2}-\frac{1}{5}(\uparrow 2) \mathbb{1}_{5}=\frac{2}{5}[2,5,7,10,12,10,7,5,2] .
$$

For $N=4$ :

$$
\begin{aligned}
h_{1,2}^{4,3} & =\mathbb{1}_{7}^{2}-\frac{1}{7}(\uparrow 2) \mathbb{1}_{7} \\
& =\frac{2}{7}[3,7,10,14,17,21,24,21,17,14,10,7,3] .
\end{aligned}
$$

Note that, as expected (by definition) the filter $h_{1,2}^{N, 3}$, and therefore $h_{1,2}^{N, 4}$, are integer-valued (up to a constant).

\section{B. Extensions with perfect reconstruction}

We now consider the existence of synthesis filters. We start by recalling the usual perfect reconstruction equations for FIR filters.

Definition 4.3: (Perfect reconstruction (PR) and complementary filter, see e.g. Strang and Nguyen [18]) The FIR filters $g, h, g^{\sim}, h^{\sim}$, with $Z$-transforms $G, H, G^{\sim}, H^{\sim}$, satisfy the perfect reconstruction property if

$$
\begin{aligned}
G(z) G^{\sim}(z)+H(z) H^{\sim}(z) & =2 z^{-\ell}, \quad \ell \in \mathbb{N} \\
H(-z) H^{\sim}(z)+G(-z) G^{\sim}(z) & =0 .
\end{aligned}
$$

Furthermore, $g$ (resp. $g^{\sim}$ ) is said to be the complementary filter of $h$ (resp. $h^{\sim}$ ) and vice versa.

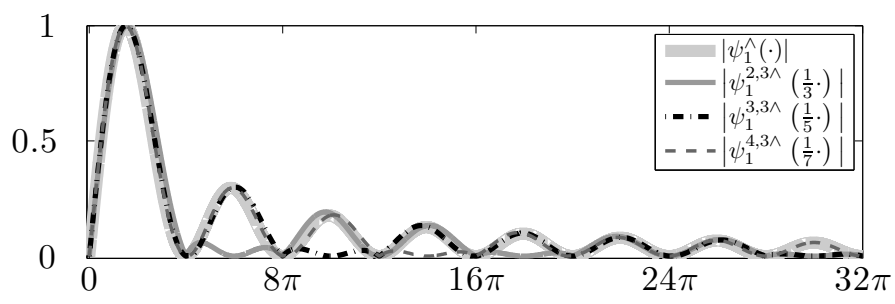

Fig. 5. The filter characteristics of the family $\psi_{M}^{N, 3}$ for $M=1$ and $N=$ $2,3,4$. We can see that $N=2$ attenuates the first harmonic, $N=3$ attenuates the second, and $N=4$ attenuates the third. 


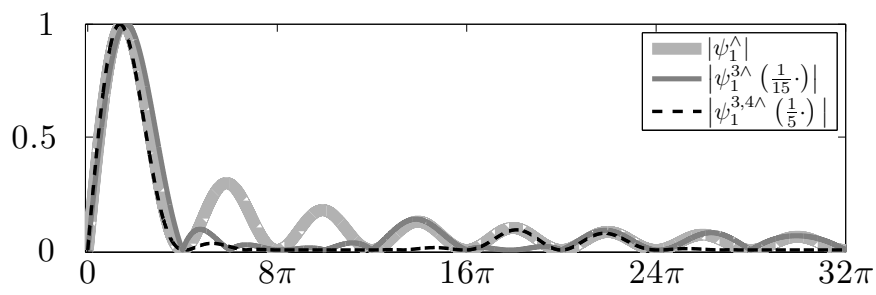

(a) $M=1, N=3$

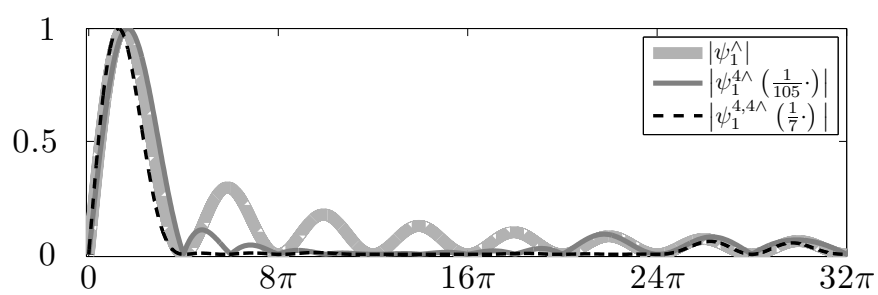

(b) $M=1, N=4$

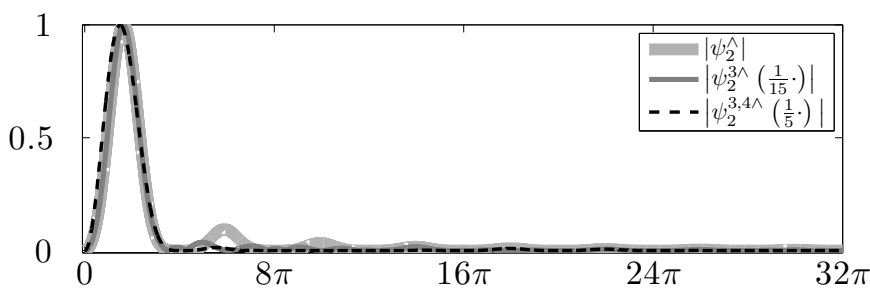

(c) $M=2, N=3$

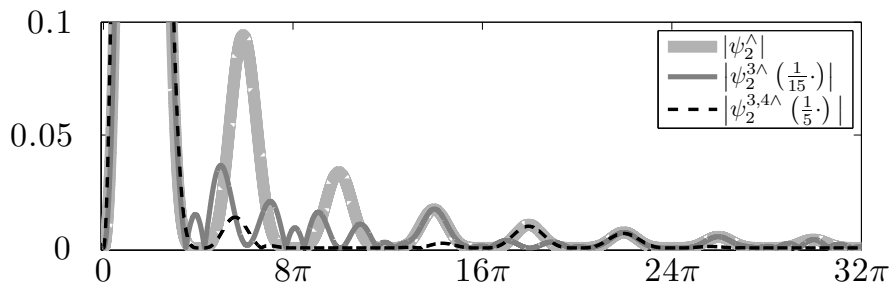

(d) $M=2, N=3$

Fig. 6. The filter characteristics of the family $\psi_{M}^{N, 4}$ for $M=1,2$ and $N=$ 3,4 compared to that of the unmodified $B$-wavelets $\psi_{M}$ and the modified wavelets $\left\{\psi_{M}^{N}\right\}$ from [14]. Plot (d) is merely a zoomed version of (c). For $\mathrm{N}$ $=3$ we see that the family $\psi_{M}^{N, 4}$ attenuates the first three harmonics. Likewise, for $N=4$, the first five harmonics are attenuated. The level of attenuation is greater than that of the family $\left\{\psi_{M}^{N}\right\}$.

Remark 4.4: Given a filter $h_{2}$, we can find its dual $h_{2}^{\tilde{2}}$ (if it exists) by solving the system of equations $(\downarrow 2)\left(h_{2} * h_{2}^{\sim}\right)[-k]=\delta_{k, 0}$, (cf. e.g. Mallat [12]).

Example 4.5: Recall, from Example 4.1, that $h_{1,2}^{2}=$ $\frac{2}{3}[1,3,4,3,1]$. Then, the dual filter is $h_{1,2}^{2}=\frac{1}{4}[-1,3,-1]$. Choosing the common alternating signs pattern (namely, in the $Z$-domain, $H_{1,3}^{2}(z):=H_{1,2}^{2}(-z)$ and $H_{1,3}^{2 \sim}(z):=$ $-H_{1,2}^{2}(-z)$ ), the complementary filter is found as $h_{1,3}^{2}=$ $\frac{1}{4}[1,3,1]$ and its dual as $h_{1,3}^{2 \sim}=\frac{2}{3}[1,-3,4,-3,1]$.

The discrete frequency responses of these filters are illustrated in Figure 8 (a). Later on, in Section $\mathrm{V}-\mathrm{B}$ a simple toy example will compare the result of applying $h_{1,2}^{2}$ and complements/duals with simply applying $h_{0}$ and $h_{1}$ and duals instead. To this end, Figure 8 (b) illustrates the effects, in the frequency domain, of the analysis/decomposition part of the

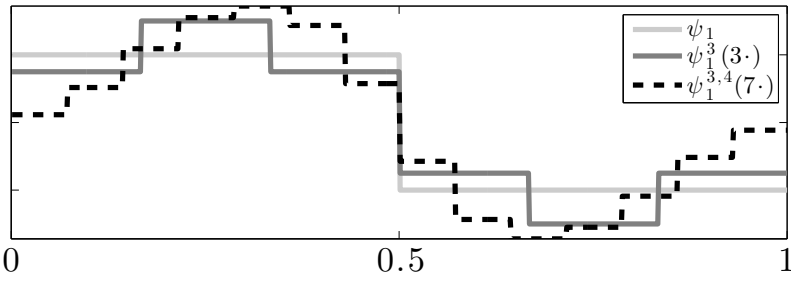

(a) $M=1$

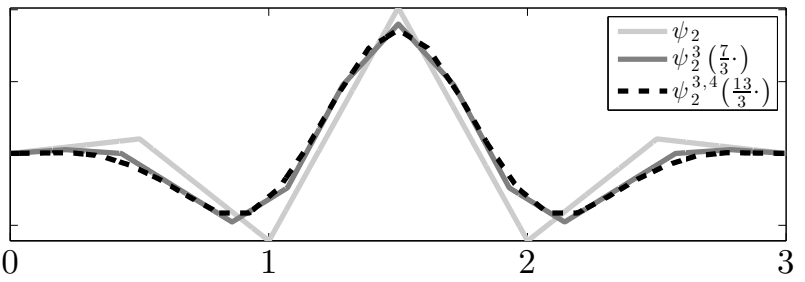

(b) $M=2$

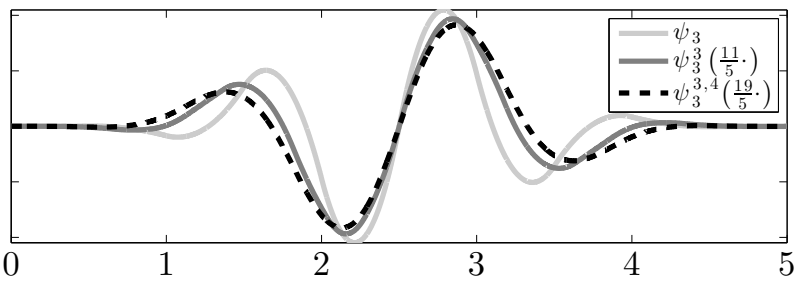

(c) $M=3$

Fig. 7. Impulse responses for the original $B$-wavelets $\psi_{M}$, and the family $\psi_{M}^{N, 4}$, for $M=1,2,3$ and $N=1,2,3$.

processing. For example, the characteristic $\left|h_{2}^{\wedge} h_{1}^{\wedge}\right|$ shows the effect of applying the filter $h_{1}$, immediately followed by $h_{1,2}^{2}$ as per the architecture defined by Figure 3

Since $h_{1,2}^{2}$ satisfies the PR conditions, we can now say that, by definition, the wavelet family $\psi_{M}^{N, 1}$ 'satisfies PR' for $N=$ 1 and for all $\mathrm{M}$ in the sense that the corresponding wavelet transform can be realised with PR filter banks (albeit in the wavelet packet architecture of Figure 3).

Proposition 4.6: (Vetterli and Herley, [21]) A filter $H(z)$ has a complementary filter if and only if $H(z)$ has no zeros in pairs at $z=z_{0}$ and $z=-z_{0}$.

Since Example 4.5 has shown that $h_{1,2}^{N}$ satisfies PR then it follows from Proposition 4.6 that the family $\psi_{M}^{N, 2}$ has PR for $N=1$ and for all $M$. Unfortunately, neither $\psi_{M}^{N, 1}$ nor $\psi_{M}^{N, 2}$ satisfies PR for $N>2$. However, we do have the following result.

Corollary 4.7: The wavelet families $\left\{\psi_{M}^{N, 3}\right\}$ and $\left\{\psi_{M}^{N, 4}\right\}$ have the PR property.

Proof We have

$$
\begin{aligned}
h_{1,2}^{N, 3} & :=\mathbb{1}_{d_{N}} *\left(\uparrow 2 d_{N}^{\sim}\right) \mathbb{1}_{1}+\gamma_{N} \mathbb{1}_{1}^{2} *(\uparrow 2) \mathbb{1}_{d_{N}} \\
& =\mathbb{1}_{d_{N}}^{2}+\gamma_{N}(\uparrow 2) \mathbb{1}_{d_{N}}
\end{aligned}
$$

Taking Z-transforms:

$$
\begin{aligned}
H_{1,2}^{N, 3}(z) & :=\left(\mathbb{1}_{d_{N}}\right)^{2}+\gamma_{N} \mathbb{1}_{d_{N}}\left(z^{2}\right) \\
& =\left(\frac{1-z^{-d_{N}}}{1-z^{-1}}\right)^{2}+\gamma_{N} \frac{1-z^{-2 d_{N}}}{1-z^{-2}}
\end{aligned}
$$


TABLE II

FILTER COEFFICIENTS FOR SOME LOW ORDER MEMBERS OF THE FAMILY $\psi_{M}^{N, 4}$ (NOTE THAT ALL FILTERS HERE ARE SYMMETRIC WITH ODD-LENGTH, $K$ SAY. HENCE ONLY THE FIRST $(K+1) / 2$ COEFFICIENTS ARE GIVEN.)

\begin{tabular}{|c|c|c|c|c|c|}
\hline \multirow[b]{2}{*}{$M, N$} & \multirow{2}{*}{$h_{M, 2}^{N, 4}$} & \multirow{2}{*}{$\tilde{h}_{M, 2}^{N, 4}$} & $M, N$ & $h_{M, 2}^{N, 4}$ & $\tilde{h}_{M, 2}^{N, 4}$ \\
\hline & & & \multirow{2}{*}{$\begin{array}{l}\mathrm{M}=2 \\
\mathrm{~N}=2\end{array}$} & \multirow{5}{*}{$\begin{array}{c}0.44444 \\
2.66667 \\
7.55556 \\
13.33333 \\
16.00000\end{array}$} & \multirow{5}{*}{$\begin{array}{l}-0.05729 \\
0.34375 \\
-0.89583 \\
1.25000\end{array}$} \\
\hline$M=1$ & 0.66667 & -0.25000 & & & \\
\hline \multirow{2}{*}{$\mathrm{N}=2$} & 2.00000 & 0.75000 & & & \\
\hline & 2.66667 & & & & \\
\hline $\mathrm{M}=1$ & 0.53333 & -0.36875 & & & \\
\hline \multirow[t]{6}{*}{$\mathrm{N}=3$} & 2.93333 & 2.02812 & $M=2$ & 0.42667 & -0.19842 \\
\hline & 8.00000 & -5.55417 & $\mathrm{~N}=3$ & 3.41333 & 1.58732 \\
\hline & 15.20000 & 10.63542 & & 13.76000 & -6.47354 \\
\hline & 23.20000 & -15.81146 & & 38.50667 & 18.50414 \\
\hline & 29.86667 & 18.15208 & & 85.65333 & -41.85599 \\
\hline & 32.53333 & & & 161.06667 & 78.57317 \\
\hline \multirow{12}{*}{$\begin{array}{l}M=1 \\
N=4\end{array}$} & 0.68571 & -126.84898 & & 263.14667 & -125.44563 \\
\hline & 331429 & 613.0342 & & 379.62667 & 173.69785 \\
\hline & 8.68571 & -1514.46740 & & 489.70667 & -211.01612 \\
\hline & $\begin{array}{l}0.00571 \\
17.94286\end{array}$ & 2873.16420 & & 569.38667 & 225.25486 \\
\hline & 32.00000 & -4783.61160 & & 598.61333 & \\
\hline & 50.17143 & 7025.56426 & $M=3$ & 0.29630 & -0.01676 \\
\hline & 71.20000 & -9316.51387 & $\mathrm{~N}=2$ & 2.66667 & 0.15088 \\
\hline & 92.68571 & 11423.22469 & & 11.55556 & -0.63379 \\
\hline & 111.0857 & -12851.8819 & & 32.00000 & 1.63037 \\
\hline & 123.8857 & 13316.5361 & & 63.11111 & -2.81917 \\
\hline & 128.6857 & & & 93.33333 & 3.38086 \\
\hline & & & & 106.07407 & \\
\hline
\end{tabular}

$$
=\frac{1-z^{-d_{N}}}{1-z^{-1}}\left(\frac{1-z^{-d_{N}}}{1-z^{-1}}+\gamma_{N} \frac{1+z^{-d_{N}}}{1+z^{-1}}\right) .
$$

Hence $H_{1,2}^{N, 3}(z)$ has roots at

$$
f_{0}(z):=\frac{1-z^{-d_{N}}}{1-z^{-1}}=0
$$

and at

$f_{1}(z):=\left(1+\gamma_{N}\right)\left(1-z^{-d_{N}-1}\right)+\left(1-\gamma_{N}\right)\left(z^{-1}-z^{-d_{N}}\right)=0$.

By Proposition 4.6 it suffices to show that $H_{1,2}^{N, 3}(z)$ does not have zeros in pairs. Assume the contrary that it does have zeros in pairs at $\left(z_{0},-z_{0}\right)$. Then so does either $f_{0}$ or $f_{1}$. But, equating $f_{0}(z)=f_{0}(-z)$ implies the contradiction $z_{0}^{-d_{N}}=0$, whereas equating $f_{1}(z)=f_{1}(-z)$, together with the fact that $d_{n}$ is odd, leads to the contradiction $z_{0}= \pm 1$. We therefore conclude that $H_{1,2}^{N, 3}(z)$ does not have zeros in pairs and that, courtesy of Vetterli and Herley (Proposition 4.6), its complementary filter exists and perfect reconstruction is possible. Since $h_{M, 2}^{N, 3}$ merely comprises autoconvolutions of $h_{1,2}^{N, 3}$ it too does not have zeros in pairs and also has PR. Likewise, since $h_{M, 2}^{N, 4}$ comprises the two factors $h_{1,2}^{N-1,3}$ and $h_{1,2}^{N, 3}$ which do not have zeros in pairs (on their own or jointly) we also conclude that $h_{M, 2}^{N, 4}$ has PR.

The main consequence of Corollary 4.7 , of course, is that Figure 3 can be realised for the wavelet families $\left\{\psi_{M}^{N, 3}\right\}$ and $\left\{\psi_{M}^{N, 4}\right\}$. Table II lists the filter coefficients of the generating FIR $h_{M, 2}^{N, 4}$ and its dual for some low-order members of the family $\left\{\psi_{M}^{N, 4}\right\}$. Note that all of these filters are symmetric with odd-length, $K$ say. Hence only the first $(K+1) / 2$ coefficients are given.

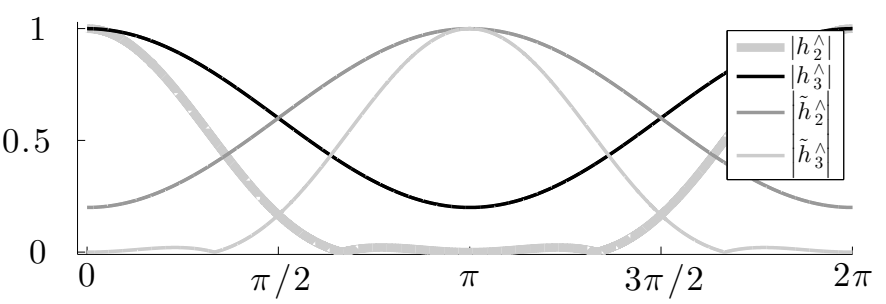

(a) Individual characteristics

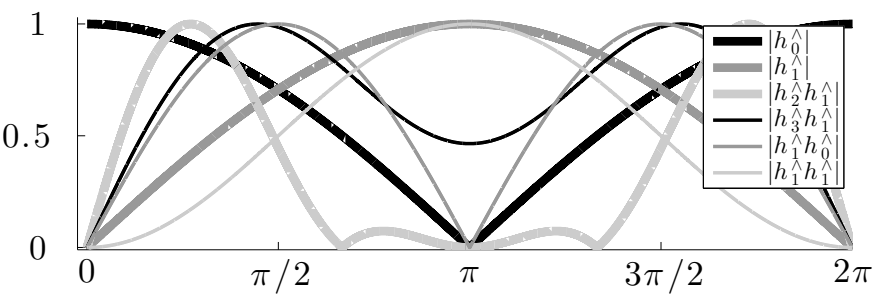

(b) Compounded characteristics

Fig. 8. Individual and compounded characteristics for the $\psi_{1}^{2,4}$ family. In (b) the proposed mixed compounded filters can be compared to the undecimated compounded Haar filters $h_{0} * h_{1}$ and $h_{1} * h_{1}$.

\section{EXAMPLES}

Although the theoretical results presented and proved here illustrate that the proposed mixed composite packet construction improves the shape of the resulting wavelet filter, it is perhaps nonetheless instructive to consider a toy example. The example presented in Section $\mathrm{V}-\mathrm{B}$ is designed to show the benefits of a filter frequency characteristic that has good mainlobe-to-sidelobe energy ratio. Intuitively, a consequence of strong harmonics in the filter is that the wavelet will pick up frequency content at higher frequencies. One simple result is that it may therefore become difficult to distinguish different frequency components. To aid the motivation, the following subsection provides a discussion of the dangers that harmonics present to poorly localised wavelet filter characteristics.

\section{A. Motivation}

For brevity of notation, we drop the $M$ and denote the $N$ th order modified wavelets by $\psi^{N}$, where $\psi^{1}$ is the original $B$-wavelet of some order of smoothness $M$. The following notation is introduced to help describe those regions of the frequency domain for which the filter characteristic is relatively large.

Definition 5.1: (Frequency domain $\lambda$-support). Define the (frequency domain) $\lambda$-support of some $\zeta \in L_{2}(\mathbb{R})$, as

$$
\Omega(\zeta ; \lambda):=\left\{\omega \in \operatorname{supp} \zeta^{\wedge}: \frac{\left|\zeta^{\wedge}(\omega)\right|}{\left|\sup \zeta^{\wedge}(\omega)\right|}>\lambda\right\}, 0 \leq \lambda \leq 1 .
$$

Note that this is extends the usual definition of support in that $\Omega(\cdot ; 0) \equiv \operatorname{supp} \cdot$. Ideally, the $\lambda$-support would lie in the set of connected intervals $\mathcal{I}$ on the real line for all $\lambda$; i.e., a bump function centred on the fundamental. However, this is not the case in practice for all but the most trivial wavelets. For the (extended) $B$-wavelet family, for instance, we have that

$$
\Omega\left(\psi^{N} ; \lambda\right)=\Omega_{N}^{0}(\lambda) \bigcup_{k \neq 0} \Omega_{N}^{k}(\lambda), \quad \Omega_{N}^{0} \in \mathcal{I}
$$


where $\Omega^{0}$ covers the frequency band region of the main lobe and $\Omega^{k}$ covers the $(2 k+1)$ th harmonic and:

$$
\Omega_{N}^{0}(\lambda) \bigcap_{k \neq 0} \Omega_{N}^{k}(\lambda) \neq \emptyset \quad \text { and } \quad \operatorname{mes} \bigcap_{k \neq 0} \Omega_{N}^{k}(\lambda) \neq 0 .
$$

Here, trivially, $\Omega\left(\psi^{N} ; \lambda\right) \notin \mathcal{I}$. If we define the set $\Lambda_{N}$ as those values of $\lambda$ such that the frequency $\lambda$-support of the $N$ th order wavelet $\psi^{N}$ is connected, namely

$$
\Lambda_{N}:=\left\{\lambda \in \mathbb{R}: \Omega\left(\psi^{N} ; \lambda\right) \in \mathcal{I}\right\},
$$

then, it follows from the preceding analysis (cf. Figure 6) that

$$
\Lambda_{1} \subset \Lambda_{2} \subset \cdots \subset \Lambda_{N} .
$$

A consequence of (11) is that $N$ controls how much the wavelet $\psi^{N}$ will suffer from interscale interference. To see this, note that, by construction, the filter characteristic of the wavelet at the $(j+\ell)$-th level $\psi_{j+\ell}^{N}$ is simply a $2^{\ell}$-dilated version of the wavelet characteristic at the $j$ th level. As such, the baseband $\lambda$-support ( $\Omega_{N}^{0}$ from Equation 10 satisfies

$$
\mathcal{D}_{\ell} \Omega_{N}^{0}\left(\psi_{j} ; \lambda\right)=\Omega_{N}^{0}\left(\psi_{j+\ell} ; \lambda\right),
$$

where the dilation operator $\mathcal{D}$ is here defined as

$$
\mathcal{D}_{\ell}(a, b):=\left(2^{-\ell} a, 2^{-\ell} b\right), \quad(a, b) \in \mathcal{I} .
$$

Consequently, a smaller $N$-order implies that there exists a larger set of $\lambda$ values for which the harmonics at scale $j$ overlap the baseband at $j+\ell$, for some $\ell$, namely:

$$
\left\{\bigcup_{k \neq 0} \mathcal{D}_{\ell} \Omega_{N}^{k}\right\} \bigcap \Omega_{N}^{0} \neq 0
$$

It is therefore possible for the frequency content of a signal to excite not only the main lobe of the wavelet filter characteristic at one level but also the harmonic lobes at other levels. Moreover, if the signal itself contains harmonics or sub-harmonics then these will both be excited at multiple scales. It is then difficult, if not impossible, to distinguish the fundamental content from one or more (sub-) harmonics. This task becomes easier as the harmonic energy in the wavelet filter characteristic is reduced (i.e., as $N$ is increased).

\section{B. Numerical experiments}

The following numerical examples are designed as simple instances of the arguments in the preceding subsection. Consider a signal with a strong frequency component at $\omega$ (Hz., say) throughout the observable time interval, together with a short-term, lower, sub-harmonic frequency at $\omega / \rho$ of smaller amplitude, plus noise, namely:

$$
f\left[t_{i}\right]=\sin \pi \omega t_{i}-\alpha \chi_{T}\left[t_{i}\right] \sin \frac{\pi \omega}{\rho} t_{i}+\epsilon_{i}, \quad \epsilon_{i} \stackrel{\mathrm{iid}}{\sim} \mathcal{N}\left(0, \sigma^{2}\right),
$$

for $\alpha<1, \rho>1, t_{i}=i / 512, i=1, \ldots, 512$, and where the characteristic, or indicator function, is defined as

$$
\chi_{T}(t):= \begin{cases}1, & t \in T \\ 0, & \text { otherwise }\end{cases}
$$

Throughout, we fix $T=[300,400] / 512$. Figures 9 through 14 illustrate the wavelet coefficient energies obtained for various parameter choices. In the leftmost column of figure 9, the Haar wavelet coefficient energies $d_{j}$ are plotted at three different scale levels. The two rightmost columns show the corresponding modified coefficients which result from applying the filters $h_{1,2}^{2,4}$ and $h_{1,3}^{2,4}$ to the undecimated Haar detail coefficients (cf. Figure 3). In other words, the fourth and fifth columns plot the proposed modified wavelet coefficients $d_{j}^{(2)}$ and $d_{j}^{(3)}$. For comparison, the second and third columns show the wavelet energies which result when we simply reuse and apply the Haar low- and high-pass filters to the undecimated $d_{j}$ instead of the proposed filters. These are denoted by ${ }_{0} d_{j}^{(2)}$ and ${ }_{0} d_{j}^{(3)}$ where the subscript 0 denotes the fact that we are applying zero-order B-wavelet filters and where the superscripts 2 and 3 denote the low- and high-passed coefficients, respectively. The black, emboldened parts of the plot indicate the location at which the extra component at $\omega / \rho \mathrm{Hz}$. is active in the signal (i.e., the interval $T$ ).

Note that there exist scale levels at which the modified coefficients tend to be relatively strong in this region compared with those that lie outside it and in the same scale level (cf. $d_{3}^{(2)}$ and $\left.d_{4}^{(2)}\right)$. The same behaviour is not as apparent when using the alternative filters ${ }_{0} d_{j}^{(2)}$ and ${ }_{0} d_{j}^{(3)}$. This indicates that the mixed composite packets are more successful at eliciting the lower frequency component, which is active in the interval $T$, from the dominant content.

This experiment is repeated in Figure 10 for different signal model parameters. Figures 11 to 14 illustrate various other experiments for the linear B-wavelet coefficients where the proposed order $M=2, N=2$ filters, namely $h_{2,2}^{2,4}$ and $h_{2,3}^{2,4}$, are compared to those of the linear B-wavelet ones ${ }_{1} d_{j}^{(2)}$ and ${ }_{1} d_{j}^{(3)}$. Again, it is seen that the weaker, lower frequency component appears to be more evident in the proposed modified wavelet coefficients. The experiments thus corroborate the preceding theory that, owing to the enhanced filter characteristic shape, the proposed modified wavelet coefficients serve as a better model identification tool than both the $B$-wavelets and the associated undecimated (and, hence, decimated) $B$-wavelet tree.

\section{CONCLUSION}

Table III offers an overview of the properties of the $B$ wavelet families introduced in this paper. It is perhaps easier to speak of the collection of families $\left\{\psi_{M}^{N, q}\right\}$ and distinguish between them using the label $q=1,2,3,4$. In the table property headings, 'multi-harmonic' refers to the ability of the wavelet family to attenuate multiple, contiguous harmonicse.g. all harmonics up to the $(N-1)$ th, say. The only family which does not have this is $q=3$. The heading 'FIR' refers to the property that the wavelet transform can be realised by the analysis part of the architecture illustrated in Figure 3, 'Decay' informally refers to the property that the filter characteristic decays sufficiently quickly (cf. Figure 4). Finally, the 'PR' heading means that the associated FIR filter $h_{2}$ has the PR property and hence a dual and complementary filter exists, cf. Definition 4.3 .

The first family, $\left\{\psi_{M}^{N}\right\}$, is merely a dilated version of the family discussed in [14]. Only the subset of wavelets 

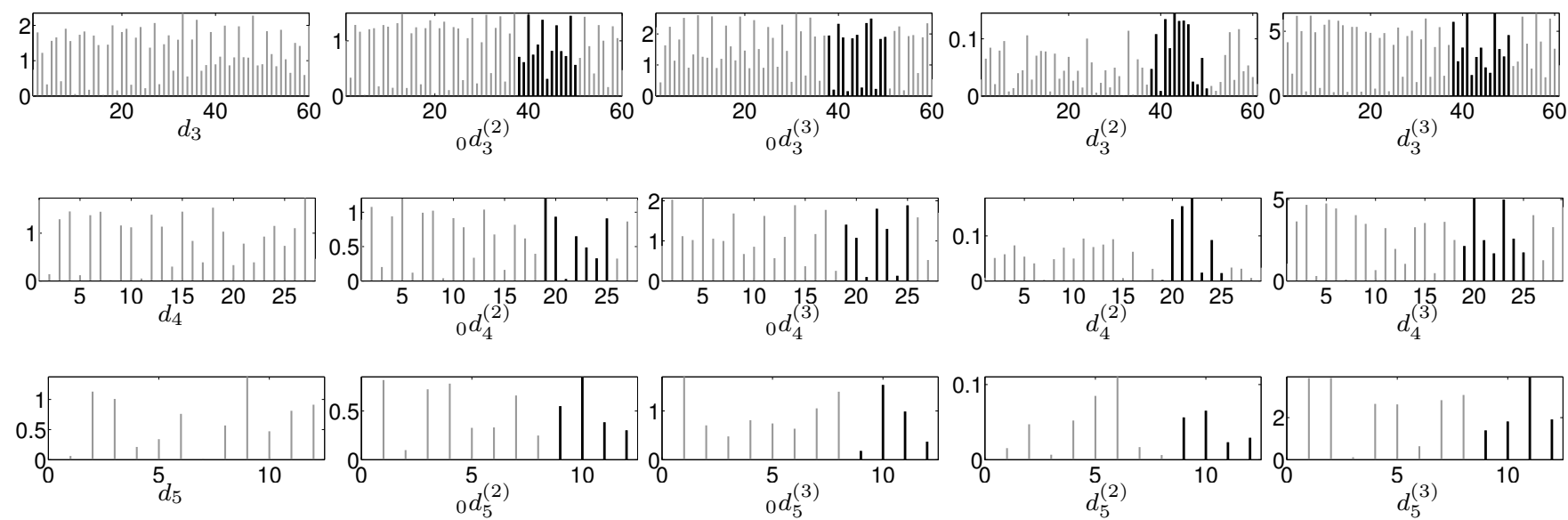

Fig. 9. Wavelet coefficients for the Haar wavelet tree with $\sigma=0.15, \alpha=0.1, \rho=3$, and $\omega=85$. From left to right: $d_{j}$ are the usual Haar wavelet coefficients; ${ }_{0} d_{j}^{(2)}$ and ${ }_{0} d_{j}^{(2)}$ denote the compounded Haar coefficients; $d_{j}^{(2)}$ and $d_{j}^{(2)}$ denote the proposed modified wavelet coefficients. The black bold part of the plots indicate the location at which the extra sub-harmonic component is active in the signal.
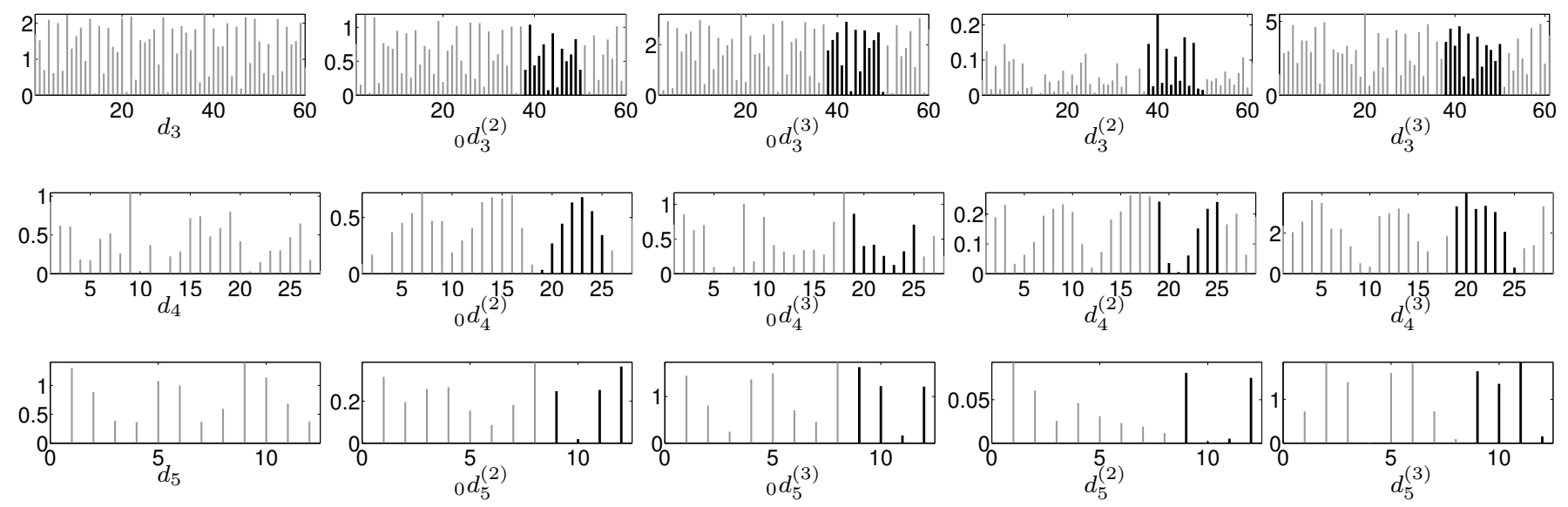

Fig. 10. Wavelet coefficients for the Haar wavelet tree with $\sigma=0.2, \alpha=0.15, \rho=3.4$, and $\omega=100$. From left to right: $d_{j}$ are the usual Haar wavelet coefficients; ${ }_{0} d_{j}^{(2)}$ and ${ }_{0} d_{j}^{(2)}$ denote the compounded Haar coefficients; $d_{j}^{(2)}$ and $d_{j}^{(2)}$ denote the proposed modified wavelet coefficients. The black bold part of the plots indicate the location at which the extra sub-harmonic component is active in the signal.

$\left\{\psi_{1}^{N}\right\}$ can be realised by an FIR filter acting on the high-pass coefficients (as in Figure 3). Likewise, PR is only possible for $M=1$ and $N \leq 2$.

The $q=1$ family fixes the FIR problem but, as Figure 4 illustrated, this fix introduces a decay issue for $M>1$. Furthermore, it only has PR for $N \leq 2$. The $q=2$ family fixes the decay but, again, PR is only possible for $N \leq 2$. It is important to note that the lack of PR does not render this family useless. Indeed, there are plenty of uses for noninvertible wavelet transforms. One quite common application is to use wavelet coefficients or functions thereof as features in machine learning classification algorithms.

The $q=3$ family satisfies the PR conditions but cannot attenuate, say, both the 1 st and 2 nd harmonic. Finally, the $q=4$ family satisfies all the properties. It can attenuate multiple, contiguous harmonics. It can be realised with FIR filters (cf. Figure 3); it has good decay and PR. All wavelets introduced here inherit many of the properties possessed by the original $B$-wavelets, namely: differentiability, number of vanishing moments, symmetry, anti-symmetry, finite support,
TABLE III

OVERVIEW OF WAVELET FAMILIES

\begin{tabular}{c|c|c|c|c} 
family & multi-harmonic & FIR & Decay & PR \\
\hline$\psi_{M}^{N}$ & $\checkmark$ & $\times$ & $\checkmark$ & $\times$ \\
\hline$\psi_{M}^{N, 1}$ & $\checkmark$ & $\checkmark$ & $\times$ & $\times$ \\
\hline$\psi_{M}^{N, 2}$ & $\checkmark$ & $\checkmark$ & $\checkmark$ & $\times$ \\
\hline$\psi_{M}^{N, 3}$ & $\times$ & $\checkmark$ & $\checkmark$ & $\checkmark$ \\
\hline$\psi_{M}^{N, 4}$ & $\checkmark$ & $\checkmark$ & $\checkmark$ & $\checkmark$ \\
\hline
\end{tabular}

and the existence of a closed form expression (for both the impulse response and filter characteristic).

The wavelet families $q=1,2,3,4$ can be realised by a wavelet packet-like architecture which contains a mixture of different FIR filters and sampling operators and allows the user to implement different combinations of wavelets according to the required mainlobe-to-sidelobe ratios and desired filter 

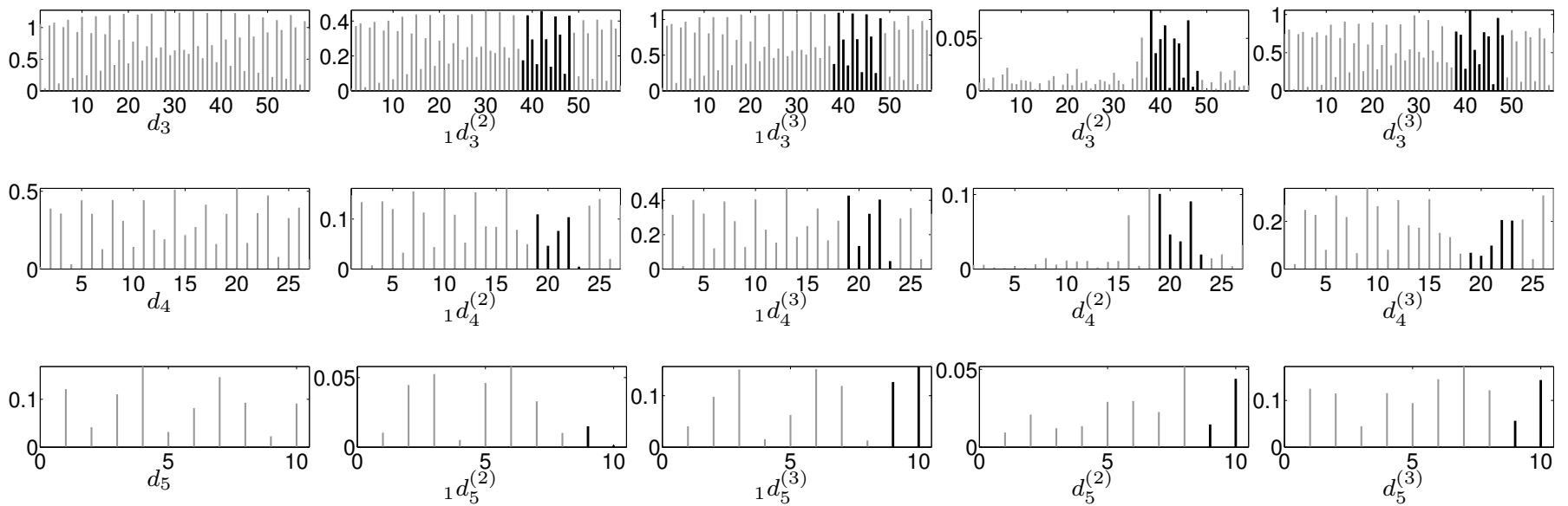

Fig. 11. Wavelet coefficients for the linear B-wavelet wavelet tree with $\sigma=0.05, \alpha=0.1, \rho=3.5$, and $\omega=85$. From left to right: $d_{j}$ are the usual Haar wavelet coefficients; ${ }_{1} d_{j}^{(2)}$ and ${ }_{1} d_{j}^{(2)}$ denote the compounded Haar coefficients; $d_{j}^{(2)}$ and $d_{j}^{(2)}$ denote the proposed modified wavelet coefficients. The black bold part of the plots indicate the location at which the extra sub-harmonic component is active in the signal.
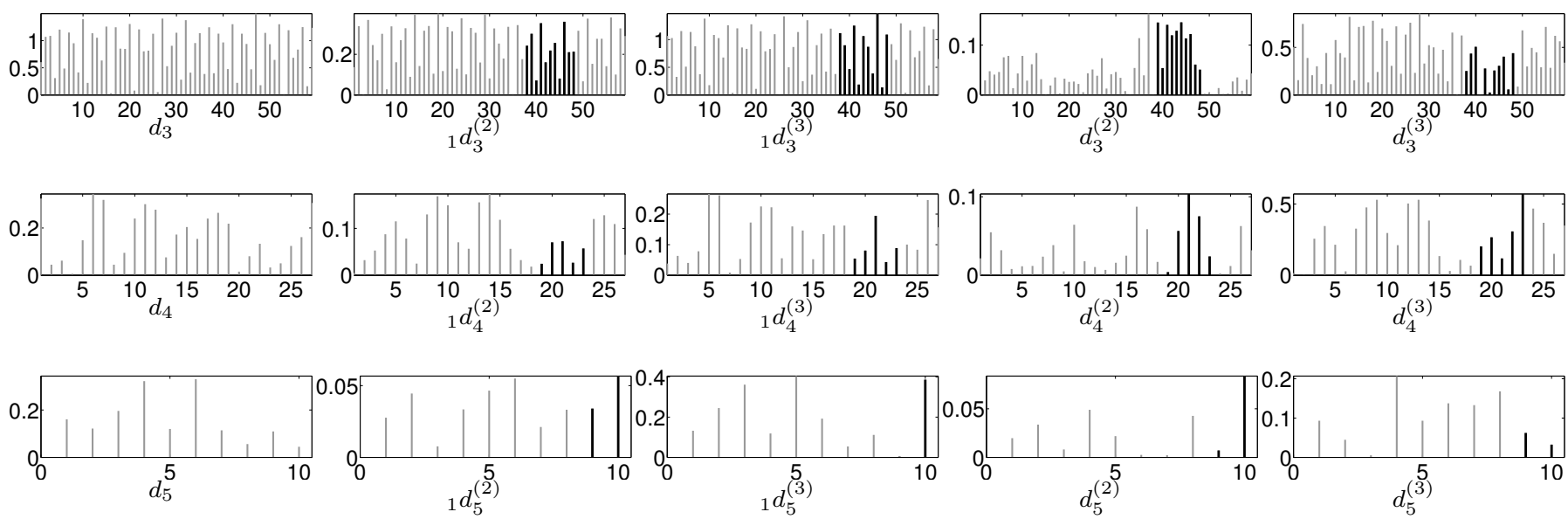

Fig. 12. Wavelet coefficients for the linear B-wavelet wavelet tree with $\sigma=0.15, \alpha=0.2, \rho=2.5$, and $\omega=90$. From left to right: $d_{j}$ are the usual Haar wavelet coefficients; ${ }_{1} d_{j}^{(2)}$ and ${ }_{1} d_{j}^{(2)}$ denote the compounded Haar coefficients; $d_{j}^{(2)}$ and $d_{j}^{(2)}$ denote the proposed modified wavelet coefficients. The black bold part of the plots indicate the location at which the extra sub-harmonic component is active in the signal.

characteristic shape. It is perhaps tempting to associate some kind of best-basis algorithm, akin to [5], [8], [10], [13], as a means of automatically adapting the modification orders $N$, given data. This, along with extensions to fractional splines and wavelets (cf. [19]) and/or complex wavelets (cf. [11], [17], [20]), may provide an interesting topic for further study.

\section{APPENDIX A \\ LEMMA 3.1}

Recall

$$
\phi_{2}\left(\frac{1}{n} \cdot\right)=\int \phi(x) \phi\left(\frac{1}{n} \cdot-x\right) \mathrm{d} x
$$

Using the identity $\phi\left(n^{-1} \cdot\right)=\sum_{k} \mathbb{1}_{n}[k] \phi(\cdot-k)$ gives

$$
\phi_{2}\left(\frac{1}{n} \cdot\right)=\sum_{k} \mathbb{1}_{n}[k] \int \phi(x) \phi(\cdot-k-n x) \mathrm{d} x .
$$

Again, using $\phi(x)=\sum_{k} \mathbb{1}_{n}[k] \phi(n x-k)$ gives

$$
\begin{aligned}
\phi_{2}\left(\frac{1}{n} \cdot\right) & =\sum_{k, \ell} \mathbb{1}_{n}[k] \mathbb{1}_{n}[\ell] \int \phi(n x-\ell) \phi(\cdot-k-n x) \mathrm{d} x \\
& =\frac{1}{n} \sum_{k, \ell} \mathbb{1}_{n}[k] \mathbb{1}_{n}[\ell-k] \int \phi(x) \phi(\cdot-\ell-x) \mathrm{d} x
\end{aligned}
$$

as stated in the main body. To complete the proof, we use a simple inductive argument by assuming the result is true for M-1. We have

$$
\begin{aligned}
\phi_{M}\left(\frac{1}{n} \cdot\right) & =\left(\phi_{M-1} * \phi\right)\left(\frac{1}{n} \cdot\right) \\
& =\int \phi(x) \phi_{M-1}\left(\frac{1}{n} \cdot-x\right) \mathrm{d} x
\end{aligned}
$$

By assumption we have that

$$
\phi_{M-1}\left(\frac{1}{n} \cdot-x\right)=\frac{1}{n^{M-2}} \sum_{k} \mathbb{1}_{n}^{M-1}[k] \phi_{M-1}(\cdot-k-n x) .
$$



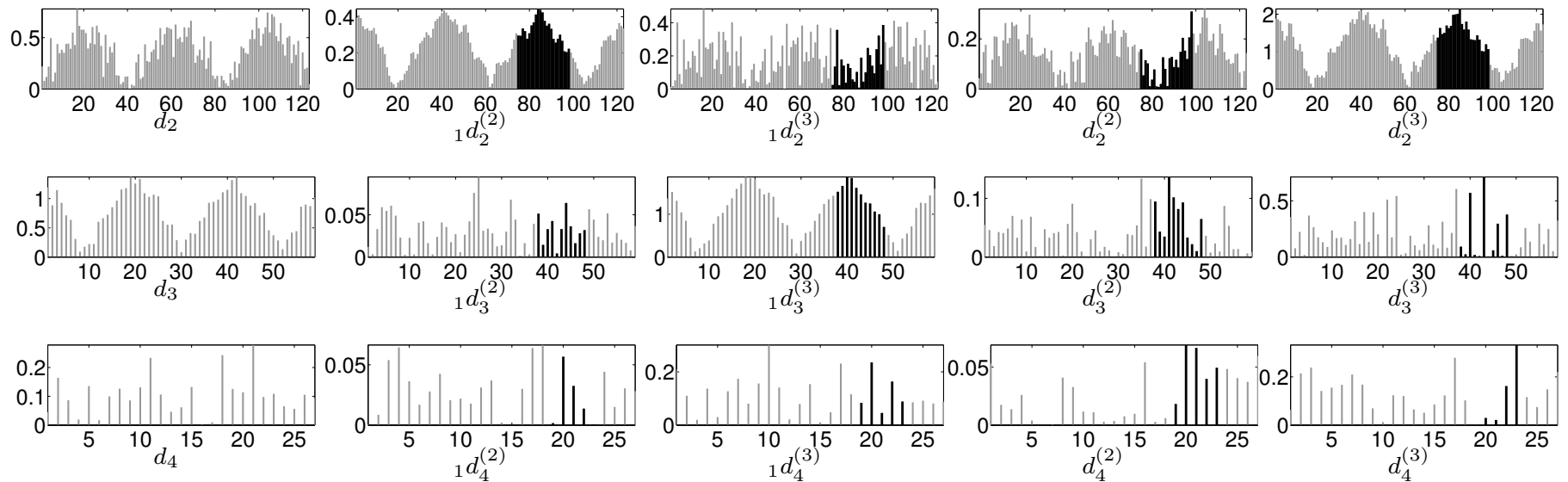

Fig. 13. Wavelet coefficients for the linear B-wavelet wavelet tree with $\sigma=0.2, \alpha=0.1, \rho=3$, and $\omega=125$. From left to right: $d_{j}$ are the usual Haar wavelet coefficients; ${ }_{1} d_{j}^{(2)}$ and ${ }_{1} d_{j}^{(2)}$ denote the compounded Haar coefficients; $d_{j}^{(2)}$ and $d_{j}^{(2)}$ denote the proposed modified wavelet coefficients. The black bold part of the plots indicate the location at which the extra sub-harmonic component is active in the signal.
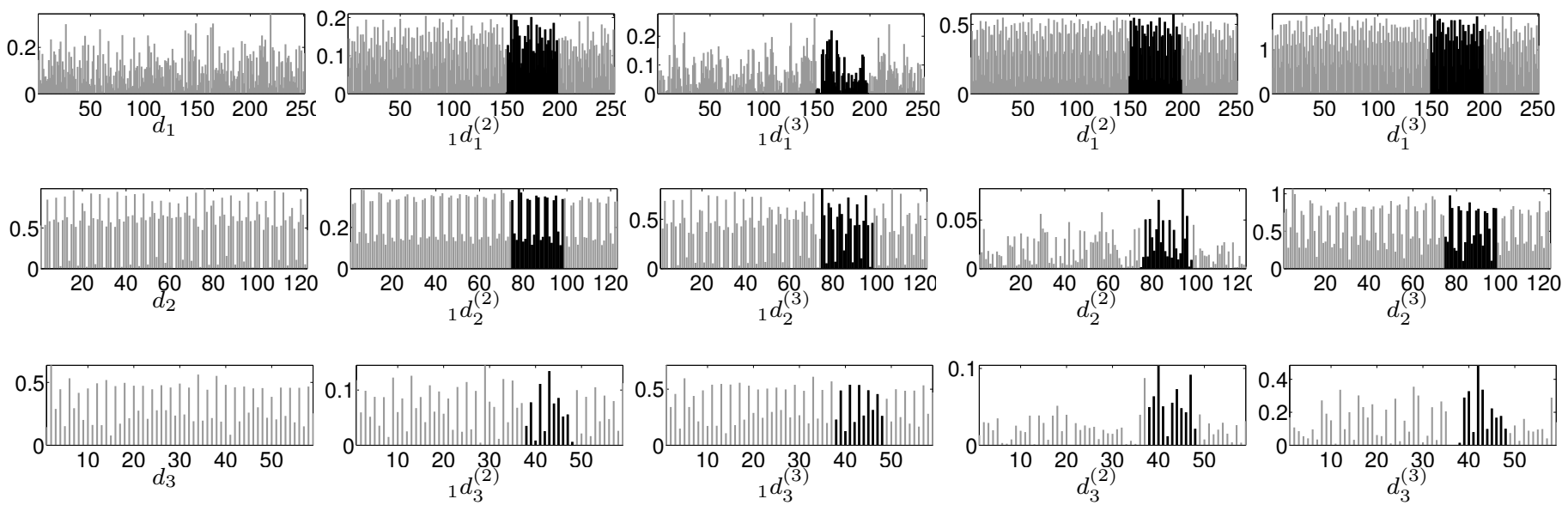

Fig. 14. Wavelet coefficients for the linear B-wavelet wavelet tree with $\sigma=0.1, \alpha=0.1, \rho=3.5$, and $\omega=160$. From left to right: $d_{j}$ are the usual Haar wavelet coefficients; ${ }_{1} d_{j}^{(2)}$ and ${ }_{1} d_{j}^{(2)}$ denote the compounded Haar coefficients; $d_{j}^{(2)}$ and $d_{j}^{(2)}$ denote the proposed modified wavelet coefficients. The black bold part of the plots indicate the location at which the extra sub-harmonic component is active in the signal.

Hence,

$\phi_{M}\left(\frac{1}{n} \cdot\right)=\frac{1}{n^{M-2}} \sum_{k} \mathbb{1}_{n}^{M-1}[k] \int \phi(x) \phi_{M-1}(\cdot-k-n x) \mathrm{d} x$.

Now, we again use $\phi(x)=\sum_{\ell} \mathbb{1}_{n}[\ell] \phi(n x-\ell)$ to get that $\phi_{M}\left(n^{-1}\right)$ is equal to

$$
\begin{aligned}
& \frac{1}{n^{M-2}} \sum_{k, \ell} \mathbb{1}_{n}[\ell] \mathbb{1}_{n}^{M-1}[k] \int \phi(n x-\ell) \phi_{M-1}(\cdot-k-n x) \mathrm{d} x \\
= & \frac{1}{n^{M-1}} \sum_{k, \ell} \mathbb{1}_{n}[\ell-k] \mathbb{1}_{n}^{M-1}[k] \int \phi(x) \phi_{M-1}(\cdot-\ell-x) \mathrm{d} x \\
= & \frac{1}{n^{M-1}} \sum_{\ell}\left(\mathbb{1}_{n} * \mathbb{1}_{n}^{M-1}\right)[\ell]\left(\phi * \phi_{M-1}\right)(\cdot-\ell)
\end{aligned}
$$

\section{REFERENCES}

[1] J. Andén and S. Mallat. Deep scattering spectrum. IEEE Transactions on Signal Processing, 62:4114-4128, 2014.

[2] A. M. Atto and Y. Berthoumieu. Wavelet packets of nonstationary random processes: contributing factors for stationarity and decorrelation. IEEE Transactions Information Theory, 58:317-330, 2012.
[3] I. Bayram and I. W. Selesnick. Frequency-domain design of overcomplete rational-dilation wavelet transforms. IEEE Transactions on Signal Processing, 57:2957-2972, 2009.

[4] A. Chebira, M. Fickus, and D. G. Mixon. Filter bank fusion frames. IEEE Transactions on Signal Processing, 59:953-963, 2011.

[5] S. S. Chen, D. L. Donoho, and M. A. Saunders. Atomic decomposition by basis pursuit. SIAM Journal on Scientific Computing, 20:33-61, 1998

[6] C. K. Chui. Wavelets: A Mathematical Tool for Signal Analysis. SIAM, 1997.

[7] C. K. Chui and J. Z. Wang. On compactly supported spline-wavelets and a duality principle. Transactions of the American Mathematical Society, 330:903-915, 1992.

[8] R. R. Coifman and M. V. Wickerhauser. Entropy-based algorithms for best basis selection. IEEE Transactions on Information Theory, 38:713$718,1992$.

[9] F. Dovis, M. Mondin, and F. Daneshgaran. The modified Gaussian: a novel wavelet with low sidelobes with applications to digital communications. IEEE Communications Letters, 2(8):208-210, 1998.

[10] P. L. Dragotti. Wavelet footprints: Theory, algorithms, and applications. IEEE Transactions on Signal Processing, 51:1306-1323, 2003.

[11] N. G. Kingsbury. Complex wavelets for shift invariant analysis and filtering of signals. Journal of Applied and Computational Harmonic Analysis, 10(3):234-253, 2001.

[12] S. Mallat. A Wavelet Tour of Signal Processing, Third Edition: The Sparse Way. Academic Press, 2008. 
[13] S. Mallat and Z. Zhang. Matching pursuit with timefrequency dictionaries. IEEE Transactions on Signal Processing, 41:3397-3415, 1993.

[14] J. D. B. Nelson. A wavelet filter enhancement scheme with a fast integral B-wavelet transform and pyramidal multi-B-wavelet algorithm. Journal of Applied and Computational Harmonic Analysis, 18:234-251, 2005.

[15] R. A. Gopinath P. Steffen, P. N. Heller and C. S. Burrus. Theory of regular m-band wavelet bases. IEEE Transactions on Signal Processing, 41:3497-3511, 1993.

[16] I. J. Schoenberg. Cardinal interpolation and spline functions. Journal of Approximation Theory, 2:167-206, 1969.

[17] I. W. Selesnick, R. G. Baraniuk, and N. G. Kingsbury. The dualtree complex wavelet transform. IEEE Signal Processing Magazine, 22(6):123-151, 2005.

[18] G. Strang and T. Nguyen. Wavelets and Filter Banks. WellesleyCambridge Press, 1996.

[19] M. Unser and T. Blu. Fractional splines and wavelets. SIAM Review, 42:43-97, 2000.

[20] M. Unser, D. Sage, and D. Van De Ville. Multiresolution monogenic signal analysis using the Riesz-Laplace wavelet transform. IEEE Transactions on Image Processing, 18:2402-2418, 2009.

[21] M. Vetterli and C. Herley. Wavelets and filter banks: Theory and design. IEEE Transactions on Signal Processing, 40:2207-2232, 1992.

[22] T. Weickert, C. Benhaminsen, and U. Kiencke. Analytic wavelet packetscombining the dual-tree approach with wavelet packets for signal analysis and filtering. IEEE Transactions on Signal Processing, 57:493502, 2009.

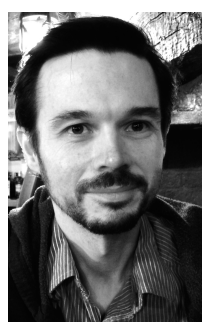

James D. B. Nelson joined the Department of Statistical Science at University College London as a Lecturer (Assistant Professor) in 2010 and became Senior Lecturer (Associate Professor) in 2013. After a PhD in applied harmonic analysis from the Mathematics Department at Anglia Polytechnic University (1998-2001), he held post-doc positions in: the Applied Mathematics and Computing Group at the University of Cranfield (2001-2004); the Information: Signals, Images, and Systems Research Group at the University of Southampton (20042006); and the Signal Processing and Communications Laboratory at the University of Cambridge (2006-2010).

Dr. Nelson's interests span interconnecting aspects of harmonic analysis, signal processing, and computational statistics. 